\title{
EXTENSIONS OF REGULARITY FOR A LÉVY PROCESS*
}

\author{
R. A. MALLER ${ }^{\dagger}$
}

\begin{abstract}
We obtain necessary and sufficient conditions for the finiteness of certain moment functions of the random variable $T_{0}^{-}$, which is the first passage time of a Lévy process $\left(X_{t}\right)_{t \geqslant 0}$ below zero, and the position $X_{T_{0}^{-}}$of the process at this time. Our results generalize classical results of Rogozin and Bertoin on the regularity of $X$, and extend earlier results of Blumenthal and Getoor on the regularity index.
\end{abstract}

Key words. regularity of a real-valued Lévy process, dominance of the positive part of a Lévy process over the negative part, first passage of a Lévy process below zero, first passage time, dominated variation conditions, Rogozin regularity condition

DOI. $10.1137 /$ S0040585X97T988824

1. Introduction. There is a long and distinguished history concerning regularity of a real-valued Lévy process $\left(X_{t}\right)_{t \geqslant 0}, X_{0}=0$, with significant contributions by Shtatland, Rogozin, and Bertoin. Let

$$
T_{0}^{-}:=\inf \left\{t>0: X_{t}<0\right\} .
$$

We say 0 is regular for $(-\infty, 0)$ at 0 if $\mathbf{P}\left(T_{0}^{-}=0\right)=1$. If this is not the case, then $\mathbf{P}\left(T_{0}^{-}=0\right)=0$ by the Blumenthal zero-one law, and we say that 0 is irregular for $(-\infty, 0)$. Rogozin $[17]$ showed that

$$
0 \text { is irregular for }(-\infty, 0) \text { if and only if } \int_{0}^{1} t^{-1} \mathbf{P}\left(X_{t} \leqslant 0\right) \mathrm{d} t<\infty .
$$

Statement (1.1) is equivalent to having $T_{0}^{-}>0$ almost surely (a.s.). Bertoin [3] replaced the integral condition in (1.1) with a more accessible integral condition on the Lévy measure of $X$. The behavior can be delineated as follows. When $X \in b v$ ( $X$ is of bounded variation), then $\lim _{t \downarrow 0} X_{t} / t=\mathrm{d}_{X}$ a.s., where $\mathrm{d}_{X}$ is the drift of $X$ (see [20]). Thus when $X \in b v$ and $\mathrm{d}_{X}<0,0$ is regular for $(-\infty, 0)$, while $\mathrm{d}_{X}>0$ implies that 0 is irregular for $(-\infty, 0)$. When $X \notin b v$, Shtatland and Rogozin (see [20], [18], and [17]) established that $\lim \sup _{t \downarrow 0} X_{t} / t=-\liminf _{t \downarrow 0} X_{t} / t=+\infty$ a.s., so 0 is regular for $(-\infty, 0)$ in this case.

That leaves the case $X \in b v$ with drift $\mathrm{d}_{X}=0$ to consider. Assuming this, Bertoin showed (see [3, Theorems 1 and 2]) that

$$
0 \text { is irregular for }(-\infty, 0) \text { if and only if } \int_{0}^{1} \bar{\Pi}^{-}(x) \mathrm{d}\left(\frac{x}{\int_{0}^{x} \bar{\Pi}^{+}(y) \mathrm{d} y}\right)<\infty
$$

$$
\text { if and only if } \lim _{t \downarrow 0} \frac{X_{t}^{(-)}}{X_{t}^{(+)}}=0 \text { a.s., }
$$

\footnotetext{
* Received by the editors June 15, 2016; accepted for publication (in revised form) July 31, 2016. This research was partially supported by ARC grant DP1092502. Originally published in the Russian journal Teoriya Veroyatnostei i ee Primeneniya, 62 (2017), pp. 719-752.

http://www.siam.org/journals/tvp/62-4/T98882.html

${ }^{\dagger}$ Research School of Finance, Actuarial Studies and Statistics, Australian National University, Canberra, Australia (Ross.Maller@anu.edu.au).
} 
where $X_{t}^{( \pm)}=\sum_{0<s \leqslant t} \Delta X_{s}^{ \pm}$and $\Delta X^{ \pm}$are the positive and negative jumps of $X$, and $\bar{\Pi}^{ \pm}$are the positive and negative tails of the Lévy measure of $X$ (see (2.1)) (and we reversed signs in Bertoin's result). Each condition in (1.2) reflects in some way the dominance of the positive part of $X$, or of its Lévy measure, over the negative part.

Herein we develop more general equivalences which can be thought of as characterizing a generalized kind of regularity, related to the finiteness of certain moment functions of $T_{0}^{-}$. These conditions involve the convergence or divergence of generalized versions of the integrals in (1.1) and (1.2) (Theorem 2.1). This analysis leads naturally to defining and characterizing a generalized version of one of the Blumenthal and Getoor [7] indices involving moments of $\left|X_{T_{0}^{-}}\right|$(Theorem 2.2).

Rogozin's proof is by random walk approximations and appealing to random walk renewal results of Spitzer [21]. Bertoin's proof is by translating a large-time random walk result of Erickson [12] to the small-time Lévy situation, requiring at certain points some quite intricate calculations. Our aim is to give a proof of the equivalence of the convergences of the integrals in (1.1) and (1.2) by direct estimation of probabilities. ${ }^{1}$ This approach allows substantial generalization along the lines indicated.

2. Main results. Let $X=\left(X_{t}\right)_{t \geqslant 0}$ be a real valued, right continuous Lévy process with $X_{0}=0$, having canonical triplet $\left(\gamma \in \mathbf{R}, \sigma^{2} \geqslant 0, \Pi\right)$, thus with characteristic function $\mathbf{E} e^{i \theta X_{t}}=e^{t \Psi(\theta)}, t \geqslant 0$, where

$$
\Psi(\theta):=i \theta \gamma-\frac{1}{2} \sigma^{2} \theta^{2}+\int_{\mathbf{R}_{*}}\left(e^{i \theta x}-1-i \theta x \mathbf{1}_{\{|x| \leqslant 1\}}\right) \Pi(\mathrm{d} x), \quad \theta \in \mathbf{R} ;
$$

$\Pi$ is a Lévy measure on $\mathbf{R}_{*}:=\mathbf{R} \backslash\{0\}$ with positive, negative, and two-sided tails

$$
\begin{aligned}
\bar{\Pi}^{+}(x):= & \Pi\{(x, \infty)\}, \quad \bar{\Pi}^{-}(x):=\Pi\{(-\infty,-x)\}, \\
& \text { and } \bar{\Pi}(x):=\bar{\Pi}^{+}(x)+\bar{\Pi}^{-}(x),
\end{aligned}
$$

for $x>0$. Define truncated mean functions for $x>0$ by

$$
\nu(x):=\gamma-\int_{x<|y| \leqslant 1} y \Pi(\mathrm{d} y)
$$

and

$$
A(x):=\gamma+\left(\bar{\Pi}^{+}(1)-\bar{\Pi}^{-}(1)\right)-\int_{x}^{1}\left(\bar{\Pi}^{+}(y)-\bar{\Pi}^{-}(y)\right) \mathrm{d} y,
$$

and truncated second moment functions by

$$
V(x):=\sigma^{2}+\int_{|y| \leqslant x} y^{2} \Pi(\mathrm{d} y) \quad \text { and } \quad U(x):=\sigma^{2}+2 \int_{0}^{x} y \bar{\Pi}(y) \mathrm{d} y .
$$

The functions $\nu(x)$ and $V(x)$ are right continuous, and $A(x)$ and $U(x)$ are continuous functions, on $(0, \infty)$. Integrating by parts gives the connections

$$
A(x):=\nu(x)+x\left(\bar{\Pi}^{+}(x)-\bar{\Pi}^{-}(x)\right) \quad \text { and } \quad U(x)=V(x)+x^{2} \bar{\Pi}(x) .
$$

\footnotetext{
${ }^{1}$ For alternative proofs of Shtatland and Bertoin's results using Vigon's [22] identities see Doney [10]. See [16] for a recent proof of the Pecherskii-Rogozin identity and related ideas.
} 
The function $A(x)$ is important for characterizing when $\lim _{t \downarrow 0} \mathbf{P}\left(X_{t} \leqslant 0\right)=0$ (see [9] and our section 4 ).

We assume throughout that

$$
\Pi\left\{\mathbf{R}_{*}\right\}=\infty .
$$

When $\Pi\left(\mathbf{R}_{*}\right)<\infty, X$ is a compound Poisson process and 0 is irregular for $(-\infty, 0)$ if and only if $X$ has drift $\mathrm{d}_{X}>0$, so we eliminate this case. Since we are only concerned with local behavior, there is no loss of generality in assuming also that $\Pi$ attributes positive mass only to a neighborhood of 0 .

Introduce a class of functions $f:(0, \infty) \mapsto(0, \infty)$ which are nonincreasing and satisfy the following kinds of dominated variation conditions: there exist $c_{ \pm}>0$, $x_{0}>0, \alpha \in[0,1)$, and $\beta>0$ such that

$$
\frac{f(x \lambda)}{f(x)} \leqslant c_{+} \lambda^{-\alpha} \text { for all } 0<\lambda \leqslant 1 \text { and } 0<x \leqslant x_{0},
$$

and

$$
0<f(0+)<\infty \quad \text { or } \quad \frac{f(x \lambda)}{f(x)} \geqslant c_{-} \lambda^{-\beta} \text { for all } 0<\lambda \leqslant 1 \text { and } 0<x \leqslant x_{0} .
$$

(Throughout, $x_{0}$ will be a small positive number. We may take $x_{0}$ to be the same in (2.7) and (2.8), and in other situations in what follows. Its existence may be part of the condition, e.g., (2.11).) Note that $f(0+)>0$, and we may have $f(0+)=\infty$.

When $f$ is regularly varying at 0 with index $-\eta, 0<\eta<1$, the uniform bounds in (2.7) and (2.8) hold with $\alpha=\eta+\delta$ and $\beta=\eta-\delta$ for all $\delta \in(0, \eta)$ (by the Potter bounds; see [6, p. 25]). For more general dominated variation conditions, see [6, p. 73]. For part of the proof we need the additional condition that $f$ can be represented as the Laplace-Stieltjes transform

$$
f(x)=\int_{0}^{\infty} e^{-p x} \mu(\mathrm{d} p), \quad x>0,
$$

of a not necessarily finite nonnegative measure $\mu(\cdot)$ on $(0, \infty)$ with $\mu(0)=0$; equivalently, by a version of Bernstein's theorem [13, XIII, section 4], $f$ is completely monotone.

An important special case which satisfies (2.7)-(2.9) is $f(x)=x^{-\alpha}, 0<\alpha<1$, $x>0$. In this case $\mu(\mathrm{d} p)=p^{\alpha-1} \mathrm{~d} p / \Gamma(\alpha), p>0$. Another important special case is when the function $f \equiv 1$ satisfies (2.7) and (2.8). For another regularly varying example take the function $f(x)=x^{-\eta}|\ln x| \mathbf{1}_{\{0<x \leqslant 1\}}$ on $(0, \infty)$, which is completely monotone, being the product of two completely monotone functions, and hence satisfies $(2.7)-(2.9)$ when $0<\eta<1$.

We can now state our first main result. Let $\Pi^{(-)}(\mathrm{d} x)=-\Pi(-\mathrm{d} x), x>0$, be the restriction of $\Pi$ to $(-\infty, 0)$. Recall that $(2.6)$ is assumed throughout.

TheOrem 2.1. Assume (2.6) is fulfilled. Suppose $f$ is a nonincreasing function satisfying (2.7) and (2.8). Then the following two conditions are equivalent:

(i)

$$
\int_{0}^{1} t^{-1} f(t) \mathbf{P}\left(X_{t} \leqslant 0\right) \mathrm{d} t<\infty
$$

Copyright (c) by SIAM. Unauthorized reproduction of this article is prohibited. 
(ii)

$$
\begin{gathered}
\sigma^{2}=0, A(x)>0 \text { for all } x \in\left(0, x_{0}\right), \\
\text { and } \int_{\left(0, x_{0}\right)}\left(\frac{x}{A(x)}\right) f\left(\frac{x}{A(x)}\right) \Pi^{(-)}(\mathrm{d} x)<\infty .
\end{gathered}
$$

Either of (2.10) or (2.11) implies $\lim _{t \downarrow 0} \mathbf{P}\left(X_{t} \leqslant 0\right)=0$ as well as $X \in b v$, and if (2.11) holds, it holds with $f \equiv 1$.

Suppose, in addition, that $f$ satisfies (2.9) and that $\int_{1}^{\infty} t^{-1} f(t) \mathrm{d} t$ is finite. Then (2.10) and (2.11) are each equivalent to

$$
\begin{aligned}
& \text { (i) } T_{0}^{-}>0 \text { a.s., when } f(0+)<\infty \text {; } \\
& \text { (ii) } \mathbf{E} f\left(T_{0}^{-}\right)<\infty \text { a.s., when } f(0+)=\infty \text {. }
\end{aligned}
$$

Remarks. The case $f \equiv 1$ in Theorem 2.1 recovers the equivalences in (1.1), (1.2) (convergence of the integral in (1.2) is equivalent to convergence of the integral in (2.11) when $f \equiv 1$; see Lemma 4.7). So we have an alternative proof of Rogozin's (and Bertoin's) original results in this case.

The case $f(x)=x^{-\alpha}, 0 \leqslant \alpha<1, x>0(f \equiv 1$ when $\alpha=0)$ requires no side conditions and gives a neat generalization of Rogozin and Bertoin's results. Note that $\lim _{x \downarrow 0} A(x) / x=\infty$ when $\lim _{t \downarrow 0} \mathbf{P}\left(X_{t} \leqslant 0\right)=0$ and (2.6) holds; see (4.6) below.

Corollary 2.1. Suppose $0 \leqslant \alpha<1$. Then the following statements are equivalent:

$$
\int_{0}^{1} t^{-1-\alpha} \mathbf{P}\left(X_{t} \leqslant 0\right) \mathrm{d} t<\infty
$$

(ii) $\sigma^{2}=0, A(x)$ is positive for all $x \in\left(0, x_{0}\right)$ and

$$
\int_{\left(0, x_{0}\right)}\left(\frac{x}{A(x)}\right)^{1-\alpha} \Pi^{(-)}(\mathrm{d} x)<\infty ;
$$

(iii) $T_{0}^{-}>0$ a.s., when $\alpha=0 ; \mathbf{E}\left(\frac{1}{\left(T_{0}^{-}\right)^{\alpha}}\right)<\infty$ when $0<\alpha<1$.

Theorem 2.1 deals with the time of passage of the process below zero. In the next theorem we consider the position of the process at its first passage below zero. When $f(0+)=\infty$ and $\mathbf{E} f\left(\left|X_{T_{0}^{-}}\right|\right)<\infty$, we understand that $T_{0}^{-}>0$ a.s.

Theorem 2.2. Let $X \in$ bv with drift $\mathrm{d}_{X}=0$. Suppose $f$ is nonincreasing on $(0, \infty)$ with $f(0+)=\infty$ and $X$ satisfies $\mathbf{E} f\left(\left|X_{T_{0}^{-}}\right|\right)<\infty$. Then

$$
\int_{(0,1)} \frac{x f(x) \Pi^{(-)}(\mathrm{d} x)}{\int_{0}^{x} \bar{\Pi}^{+}(y) \mathrm{d} y}<\infty .
$$

Conversely, if

$$
\int_{0}^{1} \bar{\Pi}^{-}(x) f(x) \mathrm{d}\left(\frac{x}{\int_{0}^{x} \bar{\Pi}^{+}(y) \mathrm{d} y}\right)<\infty
$$

then $\mathbf{E} f\left(\left|X_{T_{0}^{-}}\right|\right)<\infty$.

Copyright (c) by SIAM. Unauthorized reproduction of this article is prohibited. 
Remark. The two integrals in Theorem 2.2 converge or diverge together under very general conditions but have different convergence behavior compared to the integral in Theorem 2.1, in general. The conditions in either theorem imply $\lim _{x \downarrow 0} A(x) / x=\infty$, and convergence of either integral in Theorem 2.2 implies (2.11). When $f(t)=t^{-\alpha}, 0<\alpha<1$, Theorem 2.2 gives easily checked conditions for the finiteness of $\mathbf{E}\left(\left|X_{T_{0}^{-}}\right|^{-\alpha}\right)$, a quantity introduced by Blumenthal and Getoor [7] when studying the local behavior of Lévy processes. Blumenthal and Getoor [7, p. 501] give a sufficient ${ }^{2}$ but not very explicit condition for the finiteness of $\mathbf{E}\left(\left|X_{T_{0}^{-}}\right|^{-\alpha}\right)$. The following example illustrates the difference between the integrals in Theorem 2.2 and the integral in (2.10).

Example 1. Sato [19, Theorem 47.6] uses stable processes to give examples of situations with $T_{0}^{-}=0$ a.s. or $T_{0}^{-}>0$ a.s. (interchange $+/-$ in Sato's theorem). Here we give examples of the convergences or otherwise of the moments in Theorems 2.1 and 2.2, using the canonical measure directly.

Let $X$ be a Lévy process of bounded variation with drift $\mathrm{d}_{X}=0$, and Lévy measure satisfying, for small $x>0, \bar{\Pi}^{+}(x)=\beta x^{-1}(-\ln x)^{-\beta-1}$, where $\beta>0$, and $\Pi^{(-)}(\mathrm{d} x)=2 x^{\alpha-2}(-\ln x)^{-2} \mathrm{~d} x$, when $0 \leqslant \alpha<1$. The possible behaviors are as follows:

(i) When $\alpha=0$ : $A(x)>0$ for small $x>0$ and the integral in (2.11) with $f \equiv 1$ converges for $0<\beta<1 ; A(x)<0$ for small $x>0$ when $\beta \geqslant 1$. Thus $T_{0}^{-}>0$ a.s. for $0<\beta<1$ and $T_{0}^{-}=0$ a.s. for $\beta \geqslant 1$.

(ii) When $0<\alpha<1$ : the integral in (2.11) with $f \equiv 1$ converges for all $\beta>0$ so $T_{0}^{-}>0$ a.s. As will be seen in Lemma 4.7, when (2.11) holds and $\mathrm{d}_{X}=0$, we have $A_{-}(x)=o\left(A_{+}(x)\right)$ and $A(x) \sim A_{+}(x)$ as $x \downarrow 0$. Thus $A(x) \sim(-\ln x)^{-\beta}$ as $x \downarrow 0$, so the integral in (2.11) with $f(x)=x^{-\alpha}$ converges when $0<\beta(1-\alpha)<1$ and diverges when $\beta(1-\alpha) \geqslant 1$. Both integrals in Theorem 2.2 with $f(x)=x^{-\alpha}$ converge when $0<\beta<1$ and diverge when $\beta \geqslant 1$. So when $0<\alpha<1$ we have

$$
\begin{aligned}
& \mathbf{E}\left(\frac{1}{\left(T_{0}^{-}\right)^{\alpha}}\right)<\infty>\mathbf{E}\left(\frac{1}{\left|X_{T_{0}^{-}}\right|^{\alpha}}\right) \quad \text { for } 0<\beta<1 \\
& \mathbf{E}\left(\frac{1}{\left(T_{0}^{-}\right)^{\alpha}}\right)<\infty=\mathbf{E}\left(\frac{1}{\left|X_{T_{0}^{-}}\right|^{\alpha}}\right) \quad \text { for } 1 \leqslant \beta<\frac{1}{1-\alpha} \\
& \mathbf{E}\left(\frac{1}{\left(T_{0}^{-}\right)^{\alpha}}\right)=\infty=\mathbf{E}\left(\frac{1}{\left|X_{T_{0}^{-}}\right|^{\alpha}}\right) \quad \text { for } \beta \geqslant \frac{1}{1-\alpha}
\end{aligned}
$$

When $X \in b v$, we can define the integrals

$$
A_{ \pm}(x)=\int_{0}^{x} \bar{\Pi}^{ \pm}(y) \mathrm{d} y=x \int_{0}^{1} \bar{\Pi}^{ \pm}(x y) \mathrm{d} y, \quad x \geqslant 0 .
$$

In Example 1, when $0<\alpha<1, A(x) \sim A_{+}(x)=(-\ln x)^{-\beta}$ as $x \downarrow 0$, and the intuition is as $\beta$ decreases in magnitude, so $A_{+}(x)$ becomes larger near $0, X_{t}$ becomes "more positive" near 0 , and $T_{0}^{-}$and $\left|X_{T_{0}^{-}}\right|$become larger, though not at the same rate. This suggests that the magnitudes of $T_{0}^{-}$and $\left|X_{T_{0}^{-}}\right|$might be measured in some way by the magnitude of $A_{+}$, or the relative magnitudes of $A_{+}$and $A_{-}$; but we do not explore this further here.

\footnotetext{
${ }^{2}$ Blumenthal and Getoor gave no necessary condition. The problem seems not to have been further addressed since their time. For recent results concerning the Blumenthal and Getoor indices, see, e.g., [5], [1], [15].
} 
In what follows, we present some preliminaries concerning a compound Poisson approximation to $X$ in section 3, then give preliminary proofs in section 4 . These are then extended to the proof of Theorem 2.1 and some other results in section 5 . The proof of Theorem 2.2 will be given elsewhere.

3. Preliminary notation and lemma. Denote the jump process of $X$ by $\left(\Delta X_{t}\right)_{t \geqslant 0}$, where $\Delta X_{t}=X_{t}-X_{t-}, t>0$, with $\Delta X_{0} \equiv 0$. Let $\Delta X_{t}^{+}=\max \left(0, \Delta X_{t}\right)$ and $\Delta X_{t}^{-}=\Delta X_{t}^{+}-\Delta X_{t}, t>0$. Recall that $X$ is of bounded variation $(X \in b v)$ if $\sum_{0<s \leqslant t}\left|\Delta X_{s}\right|<\infty$ a.s. for all $t>0$ or, equivalently, if $\sigma^{2}=0$ and $\int_{|x| \leqslant 1}|x| \Pi(\mathrm{d} x)<$ $\infty$. When $X \in b v$, then $\nu(0+)$ is finite and we can define $\mathrm{d}_{X}=\nu(0+)=\gamma-$ $\int_{0<|x| \leqslant 1} x \Pi(\mathrm{d} x)$, the drift of $X$ (recall the definition of $\nu(\cdot)$ in $(2.2)$ ).

The Ito decomposition [19, Theorem 19.2] can be written in the form

$$
X_{t}=t \nu(h)+\sigma Z_{t}+X_{t}^{(S, h)}+X_{t}^{(B, h)}, \quad t \geqslant 0, \quad h>0 .
$$

Here $X_{t}^{(S, h)}$ is a compensated sum of jumps less than or equal to $h$ in modulus:

$$
X_{t}^{(S, h)}=\text { a.s. } \lim _{\varepsilon \downarrow 0}\left(\sum_{0<s \leqslant t} \Delta X_{s} \mathbf{1}_{\left\{\varepsilon<\left|\Delta X_{s}\right| \leqslant h\right\}}-t \int_{\varepsilon<|x| \leqslant h} x \Pi(\mathrm{d} x)\right),
$$

and $X_{t}^{(B, h)}$ is a sum of jumps greater than $h$ in modulus:

$$
X_{t}^{(B, h)}:=\sum_{0<s \leqslant t} \Delta X_{s} \mathbf{1}_{\left\{\left|\Delta X_{s}\right|>h\right\}}, \quad t \geqslant 0 .
$$

The process $\left(Z_{t}\right)_{t \geqslant 0}$ in (3.1) is a standard Brownian motion. All three processes $\left(Z_{t}\right)_{t \geqslant 0},\left(X_{t}^{(S, h)}\right)_{t>0}$ and $\left(X_{t}^{(B, h)}\right)_{t>0}$ are independent of one another. As shown by Sato, convergence in (3.2) is a.s., uniform on compact intervals of $t$.

When $h=1$ in (3.1), we can write $X_{t}$ as

$$
X_{t}=t \gamma+\sigma Z_{t}+X_{t}^{(J)}, \quad t \geqslant 0,
$$

where now $\left(X_{t}^{(J)}\right)_{t \geqslant 0}$ is the jump process of $X$. It satisfies

$$
X_{t}^{(J)}=X_{t}^{(S, 1)}+X_{t}^{(B, 1)}=\text { a.s. } \lim _{\varepsilon \downarrow 0} X_{t}(\varepsilon),
$$

with

$$
X_{t}(\varepsilon):=\sum_{0<s \leqslant t} \Delta X_{s} \mathbf{1}_{\left\{\left|\Delta X_{s}\right|>\varepsilon\right\}}-t \int_{\varepsilon<|x| \leqslant 1} x \Pi(\mathrm{d} x), \quad t \geqslant 0 .
$$

We construct a compound Poisson process approximation to $X$ in a standard way. Choose $\varepsilon \in(0,1)$ such that $\bar{\Pi}(\varepsilon)>0$. Let $N_{t}(\varepsilon)$ be the Poisson number of jumps $\Delta X$ exceeding $\varepsilon$ in modulus up until time $t$, with $\mathbf{E} N_{t}(\varepsilon)=t \bar{\Pi}(\varepsilon)$. Let $J_{i}(\varepsilon)$ denote the magnitudes of those jumps, $i=1,2, \ldots$. The sequence $\left(J_{i}(\varepsilon)\right)_{i \geqslant 1}$ is a sequence of independent identically distributed random variables, independent of $\left(N_{t}(\varepsilon)\right)_{t>0}$, each with distribution

$$
\frac{\Pi(\mathrm{d} x) \mathbf{1}_{\{|x|>\varepsilon\}}}{\bar{\Pi}(\varepsilon)}, \quad x \in \mathbf{R} .
$$

Copyright (c) by SIAM. Unauthorized reproduction of this article is prohibited. 
Then (3.5) can be written in the form

$$
X_{t}(\varepsilon)=\sum_{i=1}^{N_{t}(\varepsilon)} J_{i}(\varepsilon)-t \int_{\varepsilon<|x| \leqslant 1} x \Pi(\mathrm{d} x), \quad t \geqslant 0 .
$$

(Set $N_{0}(\varepsilon) \equiv 0$ and $\sum_{i=1}^{0} \equiv 0$.) The identity (3.4) expresses the jump process of $X$ as a limit of compensated compound Poisson processes. It yields the following assertion.

Lemma 3.1. Under (2.6) we have, for each $t>0$,

$$
\mathbf{P}\left(X_{t} \leqslant 0\right)=\lim _{\varepsilon \downarrow 0} \mathbf{P}\left(\sigma Z_{t}+\sum_{i=1}^{N_{t}(\varepsilon)} J_{i}(\varepsilon)+\frac{\left(N_{t}(\varepsilon)-1\right) \nu(\varepsilon)}{\bar{\Pi}(\varepsilon)} \leqslant 0, N_{t}(\varepsilon) \geqslant 1\right) .
$$

Proof of Lemma 3.1. Hold $t>0$ fixed, and write

$$
\mathbf{P}\left(X_{t} \leqslant x\right)=\lim _{\varepsilon \downarrow 0} \mathbf{P}\left(t \gamma+\sigma Z_{t}+\sum_{i=1}^{N_{t}(\varepsilon)} J_{i}(\varepsilon)-t \int_{\varepsilon<|x| \leqslant 1} x \Pi(\mathrm{d} x) \leqslant x\right), \quad x \in \mathbf{R},
$$

as follows from (3.3)-(3.7), and note that, by (2.2), for $0<\varepsilon<1$,

$$
\begin{aligned}
R_{t}(\varepsilon) & :=\frac{N_{t}(\varepsilon) \nu(\varepsilon)}{\bar{\Pi}(\varepsilon)}-t \gamma+t \int_{\varepsilon<|x| \leqslant 1} x \Pi(\mathrm{d} x) \\
& =\left(\frac{t \bar{\Pi}(\varepsilon)-N_{t}(\varepsilon)}{\bar{\Pi}(\varepsilon)}\right) \int_{\varepsilon<|x| \leqslant 1} x \Pi(\mathrm{d} x)+\gamma\left(\frac{N_{t}(\varepsilon)}{\bar{\Pi}(\varepsilon)}-t\right) \\
& =O_{\mathbf{P}}\left(\bar{\Pi}^{-1 / 2}(\varepsilon)\right) \int_{\varepsilon<|x| \leqslant 1} x \Pi(\mathrm{d} x)+o_{\mathbf{P}}(1) .
\end{aligned}
$$

The last estimate follows since $\lim _{\varepsilon \downarrow 0} \bar{\Pi}(\varepsilon)=\infty$ (by $\left.(2.6)\right)$ and $N_{t}(\varepsilon) \sim \operatorname{Poiss}(t \bar{\Pi}(\varepsilon))$ imply that $\left(t \bar{\Pi}(\varepsilon)-N_{t}(\varepsilon)\right) / \sqrt{t \bar{\Pi}(\varepsilon)}$ is asymptotically standard normal as $\varepsilon \downarrow 0$. For $0<\varepsilon<\eta<1$, the Cauchy-Schwarz inequality yields

$$
\begin{aligned}
\left|\int_{\varepsilon<|x| \leqslant 1} x \Pi(\mathrm{d} x)\right| & \leqslant\left|\int_{\varepsilon<|x| \leqslant \eta} x \Pi(\mathrm{d} x)\right|+\bar{\Pi}(\eta) \\
& \leqslant \sqrt{\bar{\Pi}(\varepsilon)} \sqrt{\int_{0<|x| \leqslant \eta} x^{2} \Pi(\mathrm{d} x)}+\bar{\Pi}(\eta) .
\end{aligned}
$$

Divide by $\sqrt{\bar{\Pi}(\varepsilon)}$, then let $\varepsilon \downarrow 0$ followed by $\eta \downarrow 0$, to see that

$$
\lim _{\varepsilon \downarrow 0} \frac{1}{\sqrt{\bar{\Pi}(\varepsilon)}} \int_{\varepsilon<|x| \leqslant 1} x \Pi(\mathrm{d} x)=0,
$$

and so the right-hand side of (3.10) is $o_{\mathbf{P}}(1)$. Thus we deduce that $R_{t}(\varepsilon) \stackrel{\mathbf{P}}{\rightarrow} 0$ as $\varepsilon \downarrow 0$.

Now by (3.9) and (3.10), $\mathbf{P}\left(X_{t}<0\right)=\lim _{\delta \downarrow 0} \mathbf{P}\left(X_{t} \leqslant-\delta\right)$ is bounded from above by

$$
\liminf _{\varepsilon \downarrow 0} \mathbf{P}\left(\sigma Z_{t}+\sum_{i=1}^{N_{t}(\varepsilon)} J_{i}(\varepsilon)+\frac{N_{t}(\varepsilon) \nu(\varepsilon)}{\bar{\Pi}(\varepsilon)} \leqslant 0\right)+\lim _{\delta \downarrow 0} \limsup _{\varepsilon \downarrow 0} \mathbf{P}\left(R_{t}(\varepsilon)>\delta\right) .
$$

Copyright (c) by SIAM. Unauthorized reproduction of this article is prohibited. 
The second term is 0 since $R_{t}(\varepsilon) \stackrel{\mathbf{P}}{\rightarrow} 0$ as $\varepsilon \downarrow 0$. On the other hand, $\mathbf{P}\left(X_{t} \leqslant 0\right)=$ $\lim _{\delta \downarrow 0} \mathbf{P}\left(X_{t} \leqslant \delta\right)$ is bounded from below by

$$
\limsup _{\varepsilon \downarrow 0} \mathbf{P}\left(\sigma Z_{t}+\sum_{i=1}^{N_{t}(\varepsilon)} J_{i}(\varepsilon)+\frac{N_{t}(\varepsilon) \nu(\varepsilon)}{\bar{\Pi}(\varepsilon)} \leqslant 0\right)-\lim _{\delta \downarrow 0} \limsup _{\varepsilon \downarrow 0} \mathbf{P}\left(R_{t}(\varepsilon)<-\delta\right) .
$$

Again the second term is equal to $0 ; X$ is not compound Poisson, so $\mathbf{P}\left(X_{t}=x\right)=0$ for $t>0$ and $x \in \mathbf{R}$ [19, Theorem 27.4], thus $\mathbf{P}\left(X_{t}<0\right)=\mathbf{P}\left(X_{t} \leqslant 0\right)$, and (3.12) and (3.13) together give (3.8) since, by (3.11), $\nu(\varepsilon)=o(\bar{\Pi}(\varepsilon))$, and $\mathbf{P}\left(N_{t}(\varepsilon)=0\right)=$ $e^{-t \bar{\Pi}(\varepsilon)} \rightarrow 0$ as $\varepsilon \downarrow 0$.

4. Preliminary results. In this section we derive the machinery needed for proving the results in section 1 . Crucial in our approach are equivalences of [9] for $\lim _{t \downarrow 0} \mathbf{P}\left(X_{t} \leqslant 0\right)=0$ and a result of [4, Theorem 2], giving that

$$
\lim _{t \downarrow 0} \mathbf{P}\left(X_{t} \leqslant 0\right)=0 \text { if and only if } \lim _{t \downarrow 0} t^{-1} \int_{0}^{t} \mathbf{P}\left(X_{s} \leqslant 0\right) \mathrm{d} s=0 .
$$

We also draw on methods of [14], where a variety of general renewal theorems for random walks is given.

Lemma 4.1. Assume the integral in (1.1) converges. Then $\sigma^{2}=0$ and

$$
\lim _{t \downarrow 0} \mathbf{P}\left(X_{t} \leqslant 0\right)=0 .
$$

Conversely, (4.2) implies $\sigma^{2}=0$.

Proof of Lemma 4.1. The integral in (1.1) cannot converge, nor can (4.2) hold, if $\sigma^{2}>0$, because then $X_{t} / \sqrt{t}$ converges in distribution to a normal random variable with mean 0 as $t \downarrow 0$, and hence $\lim _{t \downarrow 0} \mathbf{P}\left(X_{t} \leqslant 0\right)=1 / 2$. So we can eliminate this possibility. Assuming convergence of the integral in (1.1), write, for $0<t<1$,

$$
t^{-1} \int_{0}^{t} \mathbf{P}\left(X_{s} \leqslant 0\right) \mathrm{d} s \leqslant \int_{0}^{t} s^{-1} \mathbf{P}\left(X_{s} \leqslant 0\right) \mathrm{d} s \rightarrow 0 \quad \text { as } t \downarrow 0,
$$

and apply (4.1) to get (4.2). Lemma 4.1 is proved.

When (4.2) holds, results of [9] are applicable. We list those we need. Assume (4.2) holds, so $\sigma^{2}=0$, and assume that $\bar{\Pi}^{-}(0+)>0$. Then Theorem 1.1 of [9] gives that the function $A(x)$ in (2.3) is positive for all small $x, 0<x \leqslant x_{0}$, and

$$
\lim _{x \downarrow 0} \frac{A(x)}{x \bar{\Pi}^{-}(x)}=\infty .
$$

When $\bar{\Pi}^{-}(0+)=0,(4.2)$ implies that $X$ is a subordinator (possibly with a drift) and $A(x) \geqslant 0$ for all small $x[9$, Remark 1$]$. In this case we can write

$$
A(x):=\mathrm{d}_{X}+\int_{0}^{x} \bar{\Pi}^{+}(y) \mathrm{d} y,
$$

where $\mathrm{d}_{X} \geqslant 0$. Conversely, (4.3) when $\bar{\Pi}^{-}(0+)>0$ or $A(x) \geqslant 0$ for all small $x>0$ when $\bar{\Pi}^{-}(0+)=0$ implies (4.2). 
In view of $(2.6)$ we can assume $x_{0}$ so small that $\bar{\Pi}(x)>0$ for $0<x \leqslant x_{0}$. Further, when (4.2) holds, we have by Lemma 2.1 of $[9]^{3}$

$$
x^{2} \bar{\Pi}(x) \leqslant U(x) \leqslant 3 x A(x) \text { for } 0<x \leqslant x_{0}
$$

(recall (2.4)). Since (2.6) is always in force, (4.5) implies that

$$
\lim _{x \downarrow 0} \frac{A(x)}{x}=\infty
$$

and (hence) that $A(x)$ is positive for all small $x, 0<x \leqslant x_{0}$. Another useful result is the following.

Lemma 4.2. Suppose (4.5) holds. Then $0<y \leqslant x \leqslant x_{0}$ implies

$$
\frac{4 A(y)}{y} \geqslant \frac{A(x)}{x} \text {. }
$$

Proof of Lemma 4.2. For $0<y \leqslant x \leqslant x_{0}$ and $c>0$

$$
\begin{aligned}
x A(y)-c y A(x) & =x A(y)-c y A(y)-c y \int_{y}^{x}\left(\bar{\Pi}^{+}(z)-\bar{\Pi}^{-}(z)\right) \mathrm{d} z \\
& \geqslant y A(y)+(x-y) A(y)-c y A(y)-c y(x-y) \bar{\Pi}^{+}(y) \\
& =(1-c) y A(y)+(x-y)\left(A(y)-c y \bar{\Pi}^{+}(y)\right) .
\end{aligned}
$$

By (4.5), the last term is positive if $c<1 / 3$. Lemma 4.2 is proved.

Lemma 4.3. Assume (4.6) holds and choose $\delta>0$. Let $b(0)=0$, and, for $t>0$, define

$$
b(t)=b(t, \delta):=\inf \left\{0<x \leqslant x_{0}: \frac{A(x)}{x} \leqslant \frac{\delta}{t}\right\} .
$$

Then $b(t)$ satisfies $0<b(t) \leqslant x_{0}$ for all $t>0$ with $\lim _{t \downarrow 0} b(t)=0$. Also $b(t)$ is left-continuous and strictly increasing in $t$ and satisfies

$$
\frac{t A(b(t))}{b(t)}=\delta, \quad t>0
$$

The function $b(\cdot)$ has a unique inverse $b^{\leftarrow}(x):=\inf \{y: b(y) \geqslant x\}$ which is nondecreasing and continuous for $x>0$. Together, $b(\cdot)$ and $b^{\leftarrow}(\cdot)$ satisfy

$$
\begin{aligned}
b\left(b^{\leftarrow}(x)\right) & \leqslant x \leqslant b\left(b^{\leftarrow}(x)+\right), \quad b(t) \leqslant x \\
& \Longrightarrow t \leqslant b^{\leftarrow}(x), \quad t<b^{\leftarrow}(x) \Longrightarrow b(t)<x .
\end{aligned}
$$

Assume in addition that (4.7) holds. Then

$$
\frac{\delta x}{A(x)} \leqslant b^{\leftarrow}(x) \leqslant \frac{4 \delta x}{A(x)} \quad \text { for } 0<x \leqslant x_{0} .
$$

\footnotetext{
${ }^{3}$ Doney's lemma is proved under assumption (4.3), but his proof works equally well when $\bar{\Pi}^{-}(0+)=0$.
}

Copyright (c) by SIAM. Unauthorized reproduction of this article is prohibited. 
Proof of Lemma 4.3. The assumption $\lim _{x \downarrow 0} A(x) / x=\infty$ ensures that $b(\cdot)$ is well defined, and has the properties specified. The relation (4.9) is satisfied since $A(\cdot)$ is continuous. Relations (4.10) is also easily verified.

To prove (4.11), assume (4.7) and note from the definition of $b(\cdot)$ that $A(y) / y \geqslant$ $\delta / t$ for any $y \leqslant b(t)$. Choose $x>0$. Then for any $\eta>0, x \leqslant b\left(b^{\leftarrow}(x)+\eta\right)$ by (4.10), so

$$
\frac{A(x)}{x} \geqslant \frac{\delta}{b^{\leftarrow}(x)+\eta}
$$

Letting $\eta \downarrow 0$ gives the first inequality in (4.11). For the second one, use

$$
\frac{\delta}{b^{\leftarrow}(x)}=\frac{A\left(b\left(b^{\leftarrow}(x)\right)\right)}{b\left(b^{\leftarrow}(x)\right)} \geqslant \frac{A(x)}{4 x},
$$

which follows from $(4.7)$ since $(4.9)$ holds and $b\left(b^{\leftarrow}(x)\right) \leqslant x$. Lemma 4.3 is proved.

We will often use the abbreviation

$$
b_{n}(\varepsilon):=b\left(\frac{n}{\bar{\Pi}(\varepsilon)}\right)
$$

whenever $\varepsilon>0, n \geqslant 1, \bar{\Pi}(\varepsilon)>0$.

Lemma 4.4. Assume (4.6) and (using Lemma 4.3) define $b(t) \in\left(0, x_{0}\right]$ by (4.8) with $\delta>0$, and define $b_{n}(\varepsilon) \in\left(0, x_{0}\right]$ by (4.12). Then for all $\eta>0$ and $t>0$

$$
\liminf _{\varepsilon \downarrow 0} \sum_{1 \leqslant n \leqslant(1+\eta) t \bar{\Pi}(\varepsilon)} n \mathbf{P}\left(N_{t}(\varepsilon)=n\right) \mathbf{P}\left(J_{1}(\varepsilon) \leqslant-b_{n}(\varepsilon)\right) \geqslant t \bar{\Pi}^{-}(b((1+\eta) t)) .
$$

Proof of Lemma 4.4. Choose $\eta>0$ and $t>0$, and then choose $\varepsilon \in(0, b((1+\eta) t))$ so small that $t \bar{\Pi}(\varepsilon)>1$. Let $n \in[1,(1+\eta) t \bar{\Pi}(\varepsilon)]$, so $b_{n}(\varepsilon) \leqslant b((1+\eta) t)$. From (3.6) calculate

$$
\mathbf{P}\left(J_{1}(\varepsilon) \leqslant-b_{n}(\varepsilon)\right)=\frac{\bar{\Pi}^{-}\left(b_{n}(\varepsilon) \vee \varepsilon\right)}{\bar{\Pi}(\varepsilon)} \geqslant \frac{\bar{\Pi}^{-}(b((1+\eta) t))}{\bar{\Pi}(\varepsilon)} .
$$

Then the sum on the left-hand side of (4.13) is not less than

$$
\begin{aligned}
& \frac{\left.\bar{\Pi}^{-}(b(1+\eta) t)\right)}{\bar{\Pi}(\varepsilon)} \sum_{1 \leqslant n \leqslant(1+\eta) t \bar{\Pi}(\varepsilon)} n \mathbf{P}\left(N_{t}(\varepsilon)=n\right) \\
& =t \bar{\Pi}^{-}(b(1+\eta) t) \mathbf{E}\left(\frac{N_{t}(\varepsilon)}{t \bar{\Pi}(\varepsilon)} \mathbf{1}_{\left\{1 \leqslant N_{t}(\varepsilon) \leqslant(1+\eta) t \bar{\Pi}(\varepsilon)\right\}}\right) .
\end{aligned}
$$

Let $\varepsilon \downarrow 0$. Then $N_{t}(\varepsilon) / \bar{\Pi}(\varepsilon) \stackrel{\mathrm{P}}{\rightarrow} t$, so by Fatou's lemma, the expectation is greater than or equal to 1 in the limit. Hence (4.13) is fulfilled. Lemma 4.4 is proved.

Lemma 4.5. Assume (4.5) holds and (using Lemma 4.3) define $b(t) \in\left(0, x_{0}\right]$ by (4.8) with $0<\delta \leqslant 1 / 12$. Then, for $t \in(0,1)$,

$$
16 \mathbf{P}\left(X_{t} \leqslant 0\right) \geqslant t \bar{\Pi}^{-}(b(t+)) .
$$

Copyright $@$ by SIAM. Unauthorized reproduction of this article is prohibited. 
Proof of Lemma 4.5. Assume (4.5) holds and fix $t \in(0,1)$ and $\varepsilon \in(0,1)$ throughout the proof. Denote the compound Poisson term in (3.8) by $S_{N_{t}(\varepsilon)}(\varepsilon)$. Then by (3.8)

$$
\begin{aligned}
2 \mathbf{P}\left(X_{t}\right. & \leqslant 0)=2 \lim _{\varepsilon \downarrow 0} \mathbf{P}\left(\sigma Z_{t}+S_{N_{t}(\varepsilon)}(\varepsilon)+\frac{\left(N_{t}(\varepsilon)-1\right) \nu(\varepsilon)}{\bar{\Pi}(\varepsilon)} \leqslant 0\right) \\
& \geqslant \liminf _{\varepsilon \downarrow 0} \mathbf{P}\left(S_{N_{t}(\varepsilon)}(\varepsilon)+\frac{\left(N_{t}(\varepsilon)-1\right) \nu(\varepsilon)}{\bar{\Pi}(\varepsilon)} \leqslant 0, \varepsilon<b\left(\frac{N_{t}(\varepsilon)}{\bar{\Pi}(\varepsilon)}\right)\right),
\end{aligned}
$$

where the inequality follows because $Z_{t}$ is independent of the other random quantities, with $\mathbf{P}\left(Z_{t} \leqslant 0\right)=1 / 2$. Take $\varepsilon \in(0,1)$ and define

$$
\mathcal{C}(\varepsilon, t):=\left\{n \geqslant 0: \varepsilon<b_{n}(\varepsilon) \leqslant b(t)\right\} .
$$

Keeping $n \in \mathcal{C}(\varepsilon, t)$, so that $0<\varepsilon<b_{n}(\varepsilon)$, set

$$
J_{i}^{n}(\varepsilon)=\left(J_{i}(\varepsilon) \vee\left(-b_{n}(\varepsilon)\right) \wedge b_{n}(\varepsilon), \quad 1 \leqslant i \leqslant n .\right.
$$

$\left(J_{i}^{n}(\varepsilon)\right.$ need not be defined when $n \notin \mathcal{C}(\varepsilon, t)$.) We proceed to replace $\nu(\varepsilon) / \bar{\Pi}(\varepsilon)$ in (4.15) by $-\mathbf{E} J_{1}^{n}(\varepsilon)$. Recall the distribution of $J_{1}(\varepsilon)$ in (3.6). It gives, for $y>0$,

$$
\mathbf{P}\left(J_{1}(\varepsilon)>y\right)=\frac{\bar{\Pi}^{+}(\varepsilon) \mathbf{1}_{y \leqslant \varepsilon}+\bar{\Pi}^{+}(y) \mathbf{1}_{y>\varepsilon}}{\bar{\Pi}(\varepsilon)},
$$

with the same for $\mathbf{P}\left(J_{1}(\varepsilon)<-y\right)$ but with $\bar{\Pi}^{+}$replaced by $\bar{\Pi}^{-}$. Write

$$
\begin{aligned}
\bar{\Pi}(\varepsilon) \mathbf{E} J_{1}^{n}(\varepsilon) & =\bar{\Pi}(\varepsilon) \int_{0}^{b_{n}(\varepsilon)}\left(\mathbf{P}\left(J_{1}(\varepsilon)>y\right)-\mathbf{P}\left(J_{1}(\varepsilon)<-y\right)\right) \mathrm{d} y \\
& =\varepsilon\left(\bar{\Pi}^{+}(\varepsilon)-\bar{\Pi}^{-}(\varepsilon)\right)+\int_{\varepsilon}^{b_{n}(\varepsilon)}\left(\bar{\Pi}^{+}(y)-\bar{\Pi}^{-}(y)\right) \mathrm{d} y \\
& =\varepsilon\left(\bar{\Pi}^{+}(\varepsilon)-\bar{\Pi}^{-}(\varepsilon)\right)+A\left(b_{n}(\varepsilon)\right)-A(\varepsilon) \quad(\text { by }(2.3)) \\
& =A\left(b_{n}(\varepsilon)\right)-\nu(\varepsilon) \quad(\text { by }(2.5)),
\end{aligned}
$$

in which $0<\varepsilon<b_{n}(\varepsilon) \leqslant b(t) \leqslant x_{0}<1$. Thus

$$
\left.\bar{\Pi}(\varepsilon) \mathbf{E} J_{1}^{n}(\varepsilon)+\nu(\varepsilon)=A\left(b_{n}(\varepsilon)\right)=A\left(b\left(\frac{n}{\bar{\Pi}(\varepsilon)}\right)\right)=\frac{\delta b(n / \bar{\Pi}(\varepsilon))}{n / \bar{\Pi}(\varepsilon)} \quad \text { by }(4.9)\right) .
$$

This leads to

$$
(n-1)\left(\mathbf{E} J_{1}^{n}(\varepsilon)+\frac{\nu(\varepsilon)}{\bar{\Pi}(\varepsilon)}\right) \leqslant \delta b\left(\frac{n}{\bar{\Pi}(\varepsilon)}\right)=\delta b_{n}(\varepsilon) .
$$

Returning to (4.15), we can now say that

$$
\begin{aligned}
& 2 \mathbf{P}\left(X_{t} \leqslant 0\right) \\
& \quad \geqslant \liminf _{\varepsilon \downarrow 0} \sum_{n \in \mathcal{C}(\varepsilon, t)} \mathbf{P}\left(N_{t}(\varepsilon)=n\right) \mathbf{P}\left(S_{n}(\varepsilon)-(n-1) \mathbf{E} J_{1}^{n}(\varepsilon)+\delta b_{n}(\varepsilon) \leqslant 0\right) .
\end{aligned}
$$

To estimate the last probability we use an argument originally due to [11]:

$$
\begin{aligned}
\mathbf{P} & \left(\sum_{i=1}^{n} J_{i}(\varepsilon)-(n-1) \mathbf{E} J_{1}^{n}(\varepsilon) \leqslant-\delta b_{n}(\varepsilon)\right) \\
& \geqslant \mathbf{P} \bigcup_{k=1}^{n}\left\{\sum_{i=1, i \neq k}^{n} J_{i}(\varepsilon)-(n-1) \mathbf{E} J_{1}^{n}(\varepsilon) \leqslant(1-\delta) b_{n}(\varepsilon), J_{k}(\varepsilon)<-b_{n}(\varepsilon)\right\}
\end{aligned}
$$

Copyright $@$ ㅇ by SIAM. Unauthorized reproduction of this article is prohibited. 


$$
\begin{aligned}
\geqslant & n \mathbf{P}\left(J_{1}(\varepsilon)<-b_{n}(\varepsilon)\right)\left(\mathbf{P}\left(\sum_{i=1}^{n-1} J_{i}(\varepsilon)-(n-1) \mathbf{E} J_{1}^{n}(\varepsilon) \leqslant(1-\delta) b_{n}(\varepsilon)\right)\right. \\
& \left.-(n-1) \mathbf{P}\left(J_{1}(\varepsilon)<-b_{n}(\varepsilon)\right)\right)
\end{aligned}
$$

(recalling $n \in \mathcal{C}(\varepsilon, t)$ ). Further note that, on $\left\{\max _{1 \leqslant i \leqslant n-1} J_{i}(\varepsilon) \leqslant b_{n}(\varepsilon)\right\}$,

$$
J_{i}(\varepsilon) \leqslant J_{i}(\varepsilon) \vee\left(-b_{n}(\varepsilon)\right)=\left(J_{i}(\varepsilon) \vee\left(-b_{n}(\varepsilon)\right)\right) \wedge b_{n}(\varepsilon)=J_{i}^{n}(\varepsilon), \quad 1 \leqslant i \leqslant n-1 .
$$

Thus

$$
\begin{aligned}
& \mathbf{P}\left(\sum_{i=1}^{n-1} J_{i}(\varepsilon)-(n-1) \mathbf{E} J_{1}^{n}(\varepsilon) \leqslant(1-\delta) b_{n}(\varepsilon)\right) \\
& \geqslant \mathbf{P}\left(\sum_{i=1}^{n-1} J_{i}^{n}(\varepsilon)-(n-1) \mathbf{E} J_{1}^{n}(\varepsilon) \leqslant(1-\delta) b_{n}(\varepsilon), \max _{1 \leqslant i \leqslant n-1} J_{i}(\varepsilon) \leqslant b_{n}(\varepsilon)\right) \\
& \geqslant \mathbf{P}\left(\sum_{i=1}^{n-1} J_{i}^{n}(\varepsilon)-(n-1) \mathbf{E} J_{1}^{n}(\varepsilon) \leqslant(1-\delta) b_{n}(\varepsilon)\right) \\
& \quad-\mathbf{P}\left(\max _{1 \leqslant i \leqslant n-1} J_{i}(\varepsilon)>b_{n}(\varepsilon)\right) .
\end{aligned}
$$

By definition, $b_{n}(\varepsilon) \leqslant x_{0}$, so by $(4.5)$, and since $\delta \leqslant 1 / 12$,

$$
\mathbf{P}\left(\max _{1 \leqslant i \leqslant n-1} J_{i}(\varepsilon)>b_{n}(\varepsilon)\right) \leqslant(n-1) \frac{\bar{\Pi}^{+}\left(b_{n}(\varepsilon)\right)}{\bar{\Pi}(\varepsilon)} \leqslant \frac{3 n A\left(b_{n}(\varepsilon)\right)}{\bar{\Pi}(\varepsilon) b_{n}(\varepsilon)}=3 \delta \leqslant \frac{1}{4} .
$$

The first probability on the right-hand side of (4.20) is, by Chebyshev's inequality, not less than

$$
1-\frac{n \mathbf{E}\left(J_{1}^{n}(\varepsilon)\right)^{2}}{(1-\delta)^{2} b_{n}^{2}(\varepsilon)}
$$

Since $n \in \mathcal{C}(\varepsilon, t)$, so $\varepsilon<b_{n}(\varepsilon)$, we have

$$
\begin{aligned}
\bar{\Pi}(\varepsilon) \mathbf{E}\left(J_{1}^{n}(\varepsilon)\right)^{2} & =2 \bar{\Pi}(\varepsilon) \int_{0}^{b_{n}(\varepsilon)} y \mathbf{P}\left(\left|J_{1}(\varepsilon)\right|>y\right) \mathrm{d} y \\
& =2 \int_{0}^{\varepsilon} y \bar{\Pi}(\varepsilon) \mathrm{d} y+2 \int_{\varepsilon}^{b_{n}(\varepsilon)} y \bar{\Pi}(y) \mathrm{d} y \\
& \leqslant 2 \int_{0}^{b_{n}(\varepsilon)} y \bar{\Pi}(y) \mathrm{d} y=U\left(b_{n}(\varepsilon)\right) .
\end{aligned}
$$

Again by $(4.5)$ and since $b_{n}(\varepsilon) \leqslant x_{0}$, we deduce that

$$
\bar{\Pi}(\varepsilon) \mathbf{E}\left(J_{1}^{n}(\varepsilon)\right)^{2} \leqslant 3 b_{n}(\varepsilon) A\left(b_{n}(\varepsilon)\right),
$$

and hence the expression in (4.21) is at least

$$
1-\frac{3 n A\left(b_{n}(\varepsilon)\right)}{(1-\delta)^{2} b_{n}(\varepsilon) \bar{\Pi}(\varepsilon)}=1-\frac{3 \delta}{(1-\delta)^{2}} \geqslant \frac{16}{25}
$$

Copyright $@$ by SIAM. Unauthorized reproduction of this article is prohibited. 
(since $\delta \leqslant 1 / 12$ ). This gives $16 / 25-1 / 4=39 / 100$ as a lower bound for the right-hand side of (4.20), hence for the middle probability on the right-hand side of (4.19), when $n \in \mathcal{C}(\varepsilon, t)$.

The last probability on the right-hand side of (4.19) is, by (4.5), for all $n \geqslant 1$,

$$
\mathbf{P}\left(J_{1}(\varepsilon)<-b_{n}(\varepsilon)\right)=\frac{\bar{\Pi}^{-}\left(b_{n}(\varepsilon)\right)}{\bar{\Pi}(\varepsilon)} \leqslant \frac{3 A\left(b_{n}(\varepsilon)\right)}{\bar{\Pi}(\varepsilon) b_{n}(\varepsilon)}=\frac{3 \delta}{n} \leqslant \frac{1}{4 n}
$$

(since $\varepsilon<b_{n}(\varepsilon)$ and $\delta \leqslant 1 / 12$ ). So a lower bound for the right-hand side of (4.19) is $n \mathbf{P}\left(J_{1}(\varepsilon)<-b_{n}(\varepsilon)\right)(39 / 100-1 / 4)$.

For $\eta>0$ we have $b_{n}(\varepsilon) \leqslant b((1+\eta) t)$ from the definition of $\mathcal{C}(\varepsilon, t)$. Then (4.18) gives

$$
\begin{aligned}
2 \mathbf{P}\left(X_{t} \leqslant 0\right) & \geqslant \frac{14}{100} \liminf _{\varepsilon \downarrow 0} \sum_{n \in \mathcal{C}(\varepsilon, t)} \mathbf{P}\left(N_{t}(\varepsilon)=n\right) n \mathbf{P}\left(J_{1}(\varepsilon)<-b_{n}(\varepsilon)\right) \\
& \geqslant \frac{1}{8} \liminf _{\varepsilon \downarrow 0} \sum_{1 \leqslant n \leqslant(1+\eta) t \bar{\Pi}(\varepsilon)} \mathbf{P}\left(N_{t}(\varepsilon)=n\right) n \mathbf{P}\left(J_{1}(\varepsilon)<-b_{n}(\varepsilon)\right) \\
& \geqslant \frac{1}{8} t \bar{\Pi}^{-}(b((1+\eta) t)) \quad(\text { by }(4.13)) .
\end{aligned}
$$

Since $\eta>0$ is arbitrary, we arrive at (4.14). Lemma (4.5) is proved.

In the following lemma we bring in the general $f$ used in Theorems 2.1 and 2.2. The following properties are useful. For $0<x \leqslant x_{0},(2.7)$ and the monotonicity of $f$ imply

$$
\begin{aligned}
x f(x) & \leqslant \int_{0}^{x} f(y) \mathrm{d} y=x \int_{0}^{1} f(y x) \mathrm{d} y \\
& \leqslant c_{+} x f(x) \int_{0}^{1} y^{-\alpha} \mathrm{d} y=\left(\frac{c_{+}}{1-\alpha}\right) x f(x)=: c_{\alpha} x f(x),
\end{aligned}
$$

showing in particular that $\int_{0}^{1} f(x) \mathrm{d} x \leqslant c_{++}:=c_{\alpha} f(1)<\infty$.

Relations (2.8) implies $f\left(x_{0} \lambda\right) \geqslant c_{-} \lambda^{-\beta} f\left(x_{0}\right) \geqslant c_{-} f\left(x_{0}\right)$ for $0<\lambda \leqslant 1$ in the case $\beta>0$, so taking $y=x_{0} \lambda$ we see that $\inf _{0<y \leqslant x_{0}} f(y) \geqslant c_{-} f\left(x_{0}\right)>0$. In the other case, when $f(0+)>0$ in (2.8), we also have $\inf _{0<y \leqslant x_{0}} f(y)>0$.

When (2.7), (2.8), and (4.11) hold, we have, for $\delta>0$ and $0<x \leqslant x_{0}$,

$$
\begin{aligned}
\min \left(1, c_{-}(4 \delta)^{-\beta}\right) f\left(\frac{x}{A(x)}\right) & \leqslant f\left(\frac{4 \delta x}{A(x)}\right) \leqslant f\left(\frac{\delta x}{A(x)}\right) \\
& \leqslant \max \left(1, c_{+} \delta^{-\alpha}\right) f\left(\frac{x}{A(x)}\right) .
\end{aligned}
$$

Lemma 4.6. Suppose $A(x)$ is positive for $0<x \leqslant x_{0}$, (4.2) holds, and (2.7) and (2.8) are satisfied. Define $b(\cdot)$ by (4.8) with $\delta>0$. Then the convergence of

$$
\int_{0}^{1} f(t) \bar{\Pi}^{-}(b(t)) \mathrm{d} t
$$

implies the convergence of the integral in (2.11), and conversely.

Copyright (c) by SIAM. Unauthorized reproduction of this article is prohibited. 
Proof of Lemma 4.6. Assume the conditions on $A(\cdot), b(\cdot), f(\cdot)$ of the lemma are fulfilled, and proceed as follows. Equation (4.2) implies (4.5), (4.6), (4.10), and (4.11). Choose $x_{1} \in(0,1)$ such that $x / A(x) \leqslant x_{0}$ for $0<x \leqslant x_{1}$ (recall (4.6)). The integral in (4.25) exceeds

$$
\begin{aligned}
\int_{0}^{1} f(t) \int_{b(t)<x \leqslant x_{1}} \Pi^{(-)}(\mathrm{d} x) \mathrm{d} t & =\int_{\left(0, x_{1}\right]} \int_{\{t: b(t)<x\}} f(t) \mathrm{d} t \Pi^{(-)}(\mathrm{d} x) \\
& \geqslant \int_{\left(0, x_{1}\right]} \int_{t<b^{\leftarrow}(x)} f(t) \mathrm{d} t \Pi^{(-)}(\mathrm{d} x) \\
& \geqslant \int_{\left(0, x_{1}\right]} b^{\leftarrow}(x) f\left(b^{\leftarrow}(x)\right) \Pi^{(-)}(\mathrm{d} x) \quad(\text { by }(4.23)) \\
& \geqslant \delta \int_{\left(0, x_{1}\right]}\left(\frac{x}{A(x)}\right) f\left(\frac{4 \delta x}{A(x)}\right) \Pi^{(-)}(\mathrm{d} x),
\end{aligned}
$$

where we used (4.10) in the first inequality, the monotonicity of $f$ in the second inequality, and (4.11) in the third. Then by (4.24), finiteness of the integral on the left-hand side of (4.26) implies that of the integral in (2.11). The converse is proved in a similar way (but is not needed for what follows). Lemma 4.6 is proved.

For the following lemma recall the definitions of $A_{ \pm}$in (2.13). Clearly functions $A_{ \pm}(x)$ increase, while $A_{ \pm}(x) / x$ decrease, having $\operatorname{limit} \lim _{x \downarrow 0} A_{ \pm}(x) / x=\infty$ when $\bar{\Pi}^{ \pm}(0+)=\infty$.

Lemma 4.7. Suppose (2.11) holds. Then $X \in b v$ with drift $\mathrm{d}_{X} \geqslant 0$ and

$$
A(x)=\mathrm{d}_{X}+A_{+}(x)-A_{-}(x), \quad x>0 .
$$

Assume further that $\mathrm{d}_{X}=0$. Then $A_{+}(x)>0$ for all small $x>0$, and $A_{-}(x)=$ $o\left(A_{+}(x)\right)$ as $x \downarrow 0$. In either case, $\mathrm{d}_{X}>0$ or $\mathrm{d}_{X}=0$, we also have

$$
\int_{\left(0, x_{0}\right]}\left(\frac{x}{\mathrm{~d}_{X}+A_{+}(x)}\right) f\left(\frac{x}{\mathrm{~d}_{X}+A_{+}(x)}\right) \Pi^{(-)}(\mathrm{d} x)<\infty
$$

as well as (4.2), i.e., $\lim _{t \downarrow 0} \mathbf{P}\left(X_{t} \leqslant 0\right)=0$.

Conversely, suppose $X \in$ bv with drift $\mathrm{d}_{X} \geqslant 0$, and assume (4.28) holds with $A_{+}(x)>0$ for all $x>0$ when $\mathrm{d}_{X}=0$. Then (2.11) holds.

When $X \in b v$ with $\mathrm{d}_{X}=0$ and $f \equiv 1$, (4.28) holds if and only if the integral in (1.2) converges.

Proof of Lemma 4.7. Assume (2.11) holds, so (since $f$ is nonincreasing with $f\left(x_{0}>0\right)$ it holds in particular with $f \equiv 1$, and suppose it were the case that $\int_{0}^{1} \bar{\Pi}^{-}(x) \mathrm{d} x=\infty$. Then

$$
\begin{aligned}
A(x) & =\gamma+\left(\bar{\Pi}^{+}(1)-\bar{\Pi}^{-}(1)\right)-\int_{x}^{1}\left(\bar{\Pi}^{+}(y)-\bar{\Pi}^{-}(y)\right) \mathrm{d} y \\
& \leqslant \gamma+\left(\bar{\Pi}^{+}(1)-\bar{\Pi}^{-}(1)\right)+\int_{x}^{1} \bar{\Pi}^{-}(y) \mathrm{d} y \sim \int_{x}^{1} \bar{\Pi}^{-}(y) \mathrm{d} y,
\end{aligned}
$$

as $x \downarrow 0$, so (2.11) with $f \equiv 1$ implies, for arbitrary $\varepsilon>0$ and $\delta>0$ small enough,

$$
\varepsilon>\int_{0}^{\delta} \frac{x \Pi^{(-)}(\mathrm{d} x)}{\int_{x}^{1} \bar{\Pi}^{-}(y) \mathrm{d} y} \geqslant \int_{z}^{\delta} \frac{x \Pi^{(-)}(\mathrm{d} x)}{\int_{x}^{1} \bar{\Pi}^{-}(y) \mathrm{d} y} \geqslant \frac{z\left(\bar{\Pi}^{-}(z)-\bar{\Pi}^{-}(\delta)\right)}{\int_{z}^{1} \bar{\Pi}^{-}(y) \mathrm{d} y}
$$

Copyright $@$ by SIAM. Unauthorized reproduction of this article is prohibited. 
for any $0<z<\delta$. We infer from (4.29) that

$$
\lim _{z \downarrow 0} \frac{z \bar{\Pi}^{-}(z)}{\int_{z}^{1} \bar{\Pi}^{-}(y) d y}=0 .
$$

The assumption $\int_{0}^{1} \bar{\Pi}^{-}(x) \mathrm{d} x=\infty$ means that $\bar{\Pi}^{-}(0+)=\infty$. Choose $\beta \in(0,1)$ so that $\bar{\Pi}^{-}(\beta)>0$ and $\int_{\beta}^{1} \bar{\Pi}^{-}(y) \mathrm{d} y>0$. We can then integrate by parts to see that

$$
\begin{aligned}
\infty & >\int_{0}^{\beta} \frac{x \Pi^{(-)}(\mathrm{d} x)}{\int_{x}^{1} \bar{\Pi}^{-}(y) \mathrm{d} y}+\frac{\beta \bar{\Pi}^{-}(\beta)}{\int_{\beta}^{1} \bar{\Pi}^{-}(y) \mathrm{d} y} \\
& =\int_{0}^{\beta} \bar{\Pi}^{-}(x) \frac{\left(\int_{x}^{1} \bar{\Pi}^{-}(y) \mathrm{d} y+x \bar{\Pi}^{-}(x)\right) \mathrm{d} x}{\left(\int_{x}^{1} \bar{\Pi}^{-}(y) \mathrm{d} y\right)^{2}} \\
& \geqslant \int_{\eta}^{\beta} \frac{\bar{\Pi}^{-}(x) \mathrm{d} x}{\int_{x}^{1} \bar{\Pi}^{-}(y) \mathrm{d} y}=\ln \left(\frac{\int_{\eta}^{1} \bar{\Pi}^{-}(x) \mathrm{d} x}{\int_{\beta}^{1} \bar{\Pi}^{-}(x) \mathrm{d} x}\right)
\end{aligned}
$$

for any $\eta \in(0, \beta)$. The last expression tends to $\infty$ as $\eta \downarrow 0$ by our assumption that $\int_{0}^{1} \bar{\Pi}^{-}(x) \mathrm{d} x=\infty$, so this must be wrong, and hence $\int_{0}^{1} \bar{\Pi}^{-}(x) \mathrm{d} x<\infty$. But then

$$
A(x)=\gamma+\left(\bar{\Pi}^{+}(1)-\bar{\Pi}^{-}(1)\right)-\int_{x}^{1}\left(\bar{\Pi}^{+}(y)-\bar{\Pi}^{-}(y)\right) \mathrm{d} y \geqslant 0
$$

implies $\int_{0}^{1} \bar{\Pi}^{+}(x) \mathrm{d} x<\infty$. Since $\sigma^{2}=0$, we conclude $X \in b v$. Integrating by parts,

$$
A(x)=\gamma+x\left(\bar{\Pi}^{+}(x)-\bar{\Pi}^{-}(x)\right)-\int_{x<|y| \leqslant 1} y \Pi(\mathrm{d} y) \rightarrow \gamma-\int_{0<|y| \leqslant 1} y \Pi(\mathrm{d} y)=\mathrm{d}_{X}
$$

as $x \downarrow 0$, thus $A(0)=\mathrm{d}_{X}$ and

$$
\begin{aligned}
A(x) & =\mathrm{d}_{X}+x\left(\bar{\Pi}^{+}(x)-\bar{\Pi}^{-}(x)\right)+\int_{0<|y| \leqslant x} y \Pi(\mathrm{d} y) \\
& =\mathrm{d}_{X}+\int_{0}^{x}\left(\bar{\Pi}^{+}(y)-\bar{\Pi}^{-}(y)\right) \mathrm{d} y,
\end{aligned}
$$

so (4.27) holds. Since $A(x)$ is positive for all small $x$, we have $\mathrm{d}_{X} \geqslant 0$.

Assume further that $\mathrm{d}_{X}=0$ and also that $\bar{\Pi}^{-}(0+)>0$. Then $A(x) \leqslant A_{+}(x)$, and so $A_{+}(x)>0$ for $x \leqslant x_{0}$, and convergence of the integral in (2.11) with $f \equiv 1$ implies

$$
\int_{\left(0, x_{0}\right]}\left(\frac{x}{A_{+}(x)}\right) \Pi^{(-)}(\mathrm{d} x)<\infty .
$$

Convergence (4.31) implies (since the function $x / A_{+}(x)$ increases, with limit 0 as $x \downarrow 0)$

$$
\lim _{x \downarrow 0} \frac{x \bar{\Pi}^{-}(x)}{A_{+}(x)}=0 \quad \text { and } \quad \lim _{x \downarrow 0} \frac{\int_{0}^{x} y \Pi^{(-)}(\mathrm{d} y)}{A_{+}(x)}=0 .
$$

Copyright (c) by SIAM. Unauthorized reproduction of this article is prohibited. 
Integration by parts then gives

$$
\lim _{x \downarrow 0} \frac{\int_{0}^{x} \bar{\Pi}^{-}(y) \mathrm{d} y}{A_{+}(x)}=\lim _{x \downarrow 0} \frac{A_{-}(x)}{A_{+}(x)}=0,
$$

so $A_{-}(x)=o\left(A_{+}(x)\right)$, as claimed. This means $A(x) \sim A_{+}(x)$ as $x \downarrow 0$ and, in view of $(2.6), \bar{\Pi}^{+}(0+)=\infty$. Then for $0<\delta<1$ and $x$ small enough that $\delta x / A(x) \leqslant$ $x / A_{+}(x)$, by $(4.24)$ we have

$$
f\left(\frac{x}{A_{+}(x)}\right) \leqslant f\left(\frac{\delta x}{A(x)}\right) \leqslant \max \left(1, c_{+} \delta^{-\alpha}\right) f\left(\frac{x}{A(x)}\right) .
$$

Thus (2.11) implies (4.28) when $\mathrm{d}_{X}=0$ and $\bar{\Pi}^{-}(0+)=\infty$. Then (4.3) follows from

$$
\frac{A(x)}{x \bar{\Pi}^{-}(x)} \sim \frac{A_{+}(x)}{x \bar{\Pi}^{-}(x)} \rightarrow \infty \quad \text { as } x \downarrow 0,
$$

and this implies (4.2). When $\bar{\Pi}^{-}(0+)=0$ and $A(x) \geqslant 0$ for all small $x$, as holds by assumption (2.11), (4.2) holds already (as discussed following Lemma 4.1). So (4.2) holds when $\mathrm{d}_{X}=0$.

Alternatively, when $\mathrm{d}_{X}>0, \lim _{x \downarrow 0} A(x)=\mathrm{d}_{X}$ by (4.30), and

$$
X_{t}=\mathrm{d}_{X} t+\sum_{0<s \leqslant t} \Delta X_{s}^{+}-\sum_{0<s \leqslant t} \Delta X_{s}^{-},
$$

in which $\sum_{0<s \leqslant t} \Delta X_{s}^{ \pm}=o_{\mathbf{P}}(t)$ as $t \downarrow 0$ (e.g., [2, Proposition 8]). So (4.2) holds and (4.28) is implied by the case $f \equiv 1$ of (2.11).

Conversely, assume $X \in b v$ and (4.28) holds. Since $X \in b v$, this implies (4.27) as shown in (4.30), so $A(x) \rightarrow \mathrm{d}_{X}$ as $x \downarrow 0$, and hence when $\mathrm{d}_{X}>0,(2.11)$ follows from (4.28) (recall (4.24)). When $\mathrm{d}_{X}=0$ and $A_{+}(x)>0$ for small $x$, (4.32) and (4.33) follow from (4.28) with $f \equiv 1$. Hence $A(x) \sim A_{+}(x)$ as $x \downarrow 0$, so $A(x)>0$ for small $x>0$ and (2.11) follows from (4.28) again.

Additionally, when $X \in b v$ and $\mathrm{d}_{X}=0,(2.11)$ implies (4.28) and (4.32) with $A_{+}(x)>0$ for small $x>0$, so we can integrate the integral in (1.2) by parts to see that it converges. Conversely, when the integral in (1.2) converges, so that $A_{+}(x)>0$ for small $x>0$, it is easy to see that the first relation in (4.32) holds, and then integration by parts shows that (4.28) holds. Lemma 4.7 is proved.

LEMMA 4.8. Suppose the nonincreasing function $f$ satisfies (2.7) and (2.8), and (2.11) holds. Then (2.10) holds.

Proof of Lemma 4.8. By Lemma 4.7, (2.11) implies that $X \in b v$ with drift $\mathrm{d}_{X} \geqslant 0$, as well as (4.2), so we have (4.3)-(4.7) available for $x_{0}$ small enough. Fix $t \in\left(0, x_{0}\right)$ and define $b(t)$ by (4.8) with $\delta>3$. Assume $t$ is small enough for $b(2 t) \leqslant x_{0}$ and choose $\eta \in(0,1 / 2)$. Since $\bar{\Pi}(0+)=\infty$ under assumption $(2.6), \lim _{\varepsilon \downarrow 0} \mathbf{P}\left(N_{t}(\varepsilon)=0\right)=0$. In what follows, keep $\varepsilon \in(0, b(t / 2))$ and so small that $(1-\eta) t \bar{\Pi}(\varepsilon) \geqslant 1$ and $\bar{\Pi}(\varepsilon)>1 / x_{0}$. Then

$$
\mathbf{P}\left(b\left(\frac{N_{t}(\varepsilon)}{\bar{\Pi}(\varepsilon)}\right) \leqslant \varepsilon\right) \leqslant \mathbf{P}\left(b\left(\frac{t}{2}\right) \leqslant \varepsilon\right)+\mathbf{P}\left(N_{t}(\varepsilon)<\frac{t \bar{\Pi}(\varepsilon)}{2}\right) \rightarrow 0 \quad \text { as } \varepsilon \downarrow 0,
$$

Copyright (c) by SIAM. Unauthorized reproduction of this article is prohibited. 
because $N_{t}(\varepsilon) / \bar{\Pi}(\varepsilon) \stackrel{\mathbf{P}}{\rightarrow} t$ as $\varepsilon \downarrow 0$, and for the same reason, $\mathbf{P}\left(\left|N_{t}(\varepsilon)-t \bar{\Pi}(\varepsilon)\right|>\right.$ $\eta t \bar{\Pi}(\varepsilon)) \rightarrow 0$ as $\varepsilon \downarrow 0$. Recall that we denote $S_{N_{t}(\varepsilon)}(\varepsilon)=\sum_{i=1}^{N_{t}(\varepsilon)} J_{i}(\varepsilon)$, and let $A_{t}(\varepsilon)$ denote the event

$$
\left\{S_{N_{t}(\varepsilon)}(\varepsilon)+\left(N_{t}(\varepsilon)-1\right) \frac{\nu(\varepsilon)}{\bar{\Pi}(\varepsilon)} \leqslant 0, \varepsilon<b\left(\frac{N_{t}(\varepsilon)}{\bar{\Pi}(\varepsilon)}\right),\left|N_{t}(\varepsilon)-t \bar{\Pi}(\varepsilon)\right| \leqslant \eta t \bar{\Pi}(\varepsilon)\right\} .
$$

Then by (3.8) with $\sigma^{2}=0$

$$
\begin{aligned}
\mathbf{P}\left(X_{t}\right. & \leqslant 0)=\lim _{\varepsilon \downarrow 0} \mathbf{P}\left(A_{t}(\varepsilon)\right) \leqslant \limsup _{\varepsilon \downarrow 0} \mathbf{P}\left(\max _{1 \leqslant i \leqslant(1+\eta) t \bar{\Pi}(\varepsilon)} J_{i}^{-}(\varepsilon)>b((1-\eta) t)\right) \\
+ & \limsup _{\varepsilon \downarrow 0} \mathbf{P}\left(S_{N_{t}(\varepsilon)}(\varepsilon)+\left(N_{t}(\varepsilon)-1\right) \frac{\nu(\varepsilon)}{\bar{\Pi}(\varepsilon)} \leqslant 0,\right. \\
& \left.\varepsilon \vee \max _{1 \leqslant i \leqslant N_{t}(\varepsilon)} J_{i}^{-}(\varepsilon) \leqslant b\left(\frac{N_{t}(\varepsilon)}{\bar{\Pi}(\varepsilon)}\right), 1 \leqslant N_{t}(\varepsilon) \leqslant(1+\eta) t \bar{\Pi}(\varepsilon)\right) .
\end{aligned}
$$

For an upper bound on the first term on the right-hand side of (4.36), use

$$
\begin{aligned}
\mathbf{P}\left(\max _{1 \leqslant i \leqslant(1+\eta) t \bar{\Pi}(\varepsilon)} J_{i}^{-}(\varepsilon)>b((1-\eta) t)\right) & \leqslant(1+\eta) t \bar{\Pi}(\varepsilon) \mathbf{P}\left(J_{1}^{-}(\varepsilon)>b((1-\eta) t)\right) \\
& \leqslant 2 t \bar{\Pi}^{-}(b((1-\eta) t)) \quad \text { (by (3.6)). }
\end{aligned}
$$

The second probability on the right-hand side of (4.36) equals

$$
\sum_{n \in \mathcal{D}(\varepsilon, t)} \mathbf{P}\left(N_{t}(\varepsilon)=n\right) \mathbf{P}\left(S_{n}(\varepsilon)+(n-1) \frac{\nu(\varepsilon)}{\bar{\Pi}(\varepsilon)} \leqslant 0, \max _{1 \leqslant i \leqslant n} J_{i}^{-}(\varepsilon) \leqslant b_{n}(\varepsilon)\right),
$$

where, recall, $b_{n}(\varepsilon)=b(n / \bar{\Pi}(\varepsilon))$, and we set

$$
\mathcal{D}(\varepsilon, t):=\left\{n: 1<n \leqslant(1+\eta) t \bar{\Pi}(\varepsilon), \varepsilon<b_{n}(\varepsilon)\right\} .
$$

Let $i_{n}=i_{n}(\varepsilon)$ be any integer in $[1, n]$ such that $\max _{1 \leqslant j \leqslant n} J_{i}^{-}(\varepsilon) \leqslant J_{i_{n}}^{-}(\varepsilon)$, and write

$$
S_{n}(\varepsilon):=\sum_{i=1, i \neq i_{n}}^{n} J_{i}(\varepsilon)+J_{i_{n}}^{+}(\varepsilon)-J_{i_{n}}^{-}(\varepsilon) \geqslant \sum_{i=1, i \neq i_{n}}^{n} J_{i}(\varepsilon)-J_{i_{n}}^{-}(\varepsilon) .
$$

Condition on the index $i_{n}$ and $J_{i_{n}}(\varepsilon)$ and use

$$
\mathbf{P}\left(J_{i}^{-}(\varepsilon) \in \mathrm{d} y\right)=\Pi^{(-)}(\mathrm{d} y) \mathbf{1}_{\{|y|>\varepsilon\}} / \bar{\Pi}(\varepsilon)
$$

(from (3.6)) to get the bound

$$
\begin{aligned}
& \mathbf{P}\left(S_{n}(\varepsilon)+\frac{(n-1) \nu(\varepsilon)}{\bar{\Pi}(\varepsilon)} \leqslant 0, \max _{1 \leqslant i \leqslant n} J_{i}^{-}(\varepsilon) \leqslant b_{n}(\varepsilon)\right) \\
& \quad \leqslant n \int_{\left(\varepsilon, b_{n}(\varepsilon)\right]} \mathbf{P}\left(S_{n-1}(\varepsilon)+\frac{(n-1) \nu(\varepsilon)}{\bar{\Pi}(\varepsilon)} \leqslant y, \max _{1 \leqslant i \leqslant n-1} J_{i}^{-}(\varepsilon) \leqslant y\right) \frac{\Pi^{(-)}(\mathrm{d} y)}{\bar{\Pi}(\varepsilon)} .
\end{aligned}
$$

Substitute in (4.38) to get as an upper bound for the second term on the right-hand side of (4.36) $t e^{-t \bar{\Pi}(\varepsilon)}$ times

$$
\begin{aligned}
& \sum_{n+1 \in \mathcal{D}(\varepsilon, t)} \frac{(t \bar{\Pi}(\varepsilon))^{n}}{n !} \int_{\varepsilon<y \leqslant b_{n+1}(\varepsilon)} \mathbf{P}\left(S_{n}(\varepsilon)+\frac{n \nu(\varepsilon)}{\bar{\Pi}(\varepsilon)} \leqslant y, \max _{1 \leqslant i \leqslant n} J_{i}^{-}(\varepsilon) \leqslant y\right) \\
& \times \Pi^{(-)}(\mathrm{d} y) .
\end{aligned}
$$

Copyright (c) by SIAM. Unauthorized reproduction of this article is prohibited. 
Notice that

$$
b_{n}(\varepsilon)=b\left(\frac{n}{\bar{\Pi}(\varepsilon)}\right) \leqslant b((1+\eta) t) \leqslant b(2 t) \leqslant x_{0},
$$

when $n \in \mathcal{D}(\varepsilon, t)$, by our choice of $t$. So $y \leqslant x_{0}$ in (4.39). By the definition of $b(\cdot)$ in (4.8), $y \leqslant b_{n+1}(\varepsilon)=b((n+1) / \bar{\Pi}(\varepsilon))$ implies

$$
\frac{A(y)}{y} \geqslant \frac{\delta}{(n+1) / \bar{\Pi}(\varepsilon)} \geqslant \frac{3 \bar{\Pi}(\varepsilon)}{n+1} \geqslant \frac{2 \bar{\Pi}(\varepsilon)}{n}
$$

(since we took $\delta>3$ ). Thus $y \leqslant n A(y) / 2 \bar{\Pi}(\varepsilon)$. Now on $\left\{\max _{1 \leqslant i \leqslant n} J_{i}^{-}(\varepsilon) \leqslant y\right\}$,

$$
S_{n}(\varepsilon)=\sum_{i=1}^{n} J_{i}(\varepsilon)=\sum_{i=1}^{n} J_{i}(\varepsilon) \vee(-y) \geqslant \sum_{i=1}^{n}\left(J_{i}(\varepsilon) \wedge y\right) \vee(-y)=S_{n}^{y}(\varepsilon),
$$

where $S_{n}^{y}(\varepsilon):=\sum_{i=1}^{n} J_{i}^{y}(\varepsilon)$ and the $J_{i}^{y}(\varepsilon)$ are independent identically distributed with the distribution of $\left(J_{1}(\varepsilon) \vee(-y)\right) \wedge y, y>0$. So we can write

$$
\begin{aligned}
& \mathbf{P}\left(S_{n}(\varepsilon)+\frac{n \nu(\varepsilon)}{\bar{\Pi}(\varepsilon)} \leqslant y, \max _{1 \leqslant j \leqslant n} J_{i}^{-}(\varepsilon) \leqslant y\right) \\
& \quad \leqslant \mathbf{P}\left(S_{n}^{y}(\varepsilon)-\mathbf{E} S_{n}^{y}(\varepsilon) \leqslant y-\mathbf{E} S_{n}^{y}(\varepsilon)-\frac{n \nu(\varepsilon)}{\bar{\Pi}(\varepsilon)}\right) .
\end{aligned}
$$

We have $X_{t} \in b v$ and $\mathrm{d}_{X} \geqslant 0$, and the same calculation as in (4.16) gives, for $y>\varepsilon$,

$$
\mathbf{E} J_{1}^{y}(\varepsilon)=\frac{A(y)-\nu(\varepsilon)}{\bar{\Pi}(\varepsilon)}
$$

so

$$
\mathbf{E} S_{n}^{y}(\varepsilon)+\frac{n \nu(\varepsilon)}{\bar{\Pi}(\varepsilon)}=n\left(\mathbf{E} J_{1}^{y}(\varepsilon)+\frac{\nu(\varepsilon)}{\bar{\Pi}(\varepsilon)}\right)=\frac{n A(y)}{\bar{\Pi}(\varepsilon)} .
$$

Thus, via (4.40), and since $y \leqslant n A(y) / 2 \bar{\Pi}(\varepsilon)$, the integrand in (4.39) is bounded by

$$
\mathbf{P}\left(S_{n}^{y}(\varepsilon)-\mathbf{E} S_{n}^{y}(\varepsilon) \leqslant-\frac{n}{2} \frac{A(y)}{\bar{\Pi}(\varepsilon)}\right) .
$$

Let $u=n A(y) / 2 \bar{\Pi}(\varepsilon)>0$. The same calculation as in (4.22) gives, for $\varepsilon<y \leqslant x_{0}$,

$$
\begin{aligned}
s_{n}^{2} & :=\operatorname{Var}\left(S_{n}^{y}(\varepsilon)\right) \leqslant n \mathbf{E}\left(J_{1}^{y}(\varepsilon)\right)^{2}=\frac{n U(y)}{\bar{\Pi}(\varepsilon)} \\
& \leqslant \frac{3 n y A(y)}{\bar{\Pi}(\varepsilon)} \quad\left(\text { by }(4.5), \text { and since } y \leqslant x_{0}\right) .
\end{aligned}
$$

Hence

$$
s_{n}^{2}+2 y u \leqslant \frac{3 n y A(y)}{\bar{\Pi}(\varepsilon)}+\frac{y n A(y)}{\bar{\Pi}(\varepsilon)}=\frac{4 n y A(y)}{\bar{\Pi}(\varepsilon)} .
$$

Copyright $@$ by SIAM. Unauthorized reproduction of this article is prohibited. 
Bernstein's inequality (e.g., [8, Exercise 4.3.14]) implies

$$
\begin{aligned}
\mathbf{P}\left(S_{n}^{y}(\varepsilon)-\mathbf{E} S_{n}^{y}(\varepsilon) \leqslant-u\right) & \leqslant \exp \left(-\frac{u^{2}}{2\left(s_{n}^{2}+2 y u\right)}\right) \\
& \leqslant \exp \left(-\frac{u^{2} \bar{\Pi}(\varepsilon)}{8 n y A(y)}\right)=\exp \left(-\frac{n A(y)}{32 y \bar{\Pi}(\varepsilon)}\right) .
\end{aligned}
$$

Now consider two cases.

Case (a). First suppose $\mathrm{d}_{X}=0$. Then by Lemma 4.7, $A_{+}(x)>0$ for $0<x \leqslant x_{0}$ and $A(x) \sim A_{+}(x)$ as $x \downarrow 0$, so we can assume $A(y) \geqslant A_{+}(y) / 2$ for $y \leqslant x_{0}$. Hence by $(4.43)$

$$
\mathbf{P}\left(S_{n}^{y}(\varepsilon)-\mathbf{E} S_{n}^{y}(\varepsilon) \leqslant-u\right) \leqslant \exp \left(-\frac{n A_{+}(y)}{64 y \bar{\Pi}(\varepsilon)}\right)
$$

Thus, defining

$$
\rho(\varepsilon, y)=\exp \left(-\frac{A_{+}(y)}{64 y \bar{\Pi}(\varepsilon)}\right)
$$

via (4.42) an upper bound for (4.39) is

$$
\begin{gathered}
t e^{-t \bar{\Pi}(\varepsilon)} \sum_{n \geqslant 0} \frac{(t \bar{\Pi}(\varepsilon))^{n}}{n !} \int_{\varepsilon<y \leqslant 1} \rho^{n}(\varepsilon, y) \Pi^{(-)}(\mathrm{d} y) \\
=t \int_{\varepsilon<y \leqslant 1} e^{-t \bar{\Pi}(\varepsilon)(1-\rho(\varepsilon, y))} \Pi^{(-)}(\mathrm{d} y) .
\end{gathered}
$$

Thus, this constitutes an upper bound for the second term on the right-hand side of (4.36), which, together with (4.37), gives an upper bound for $\mathbf{P}\left(X_{t} \leqslant 0\right)$.

Now recall the definition of the event $A_{t}(\varepsilon)$ in (4.35) and use (4.36) to write

$$
\begin{aligned}
\int_{0}^{x_{0}} & t^{-1} f(t) \mathbf{P}\left(X_{t} \leqslant 0\right) \mathrm{d} t=\int_{0}^{x_{0}} t^{-1} f(t) \liminf _{\varepsilon \downarrow 0} \mathbf{P}\left(A_{t}(\varepsilon)\right) \mathrm{d} t \\
& \leqslant \liminf _{\varepsilon \downarrow 0} \int_{0}^{x_{0}} t^{-1} f(t) \mathbf{P}\left(A_{t}(\varepsilon)\right) \mathrm{d} t \leqslant \limsup _{\varepsilon \downarrow 0} \int_{0}^{x_{0}} t^{-1} f(t) \mathbf{P}\left(A_{t}(\varepsilon)\right) \mathrm{d} t .
\end{aligned}
$$

Then from (4.36), (4.37), and (4.45), a bound for the right-hand side of (4.46) is

$$
\begin{array}{rl}
2 \int_{0}^{x_{0}} & f(t) \bar{\Pi}^{-}(b((1-\eta) t)) \mathrm{d} t \\
& +\limsup _{\varepsilon \downarrow 0} \int_{0}^{x_{0}} f(t) \int_{\varepsilon<y \leqslant 1} e^{-t \bar{\Pi}(\varepsilon)(1-\rho(\varepsilon, y))} \Pi^{(-)}(\mathrm{d} y) \mathrm{d} t .
\end{array}
$$

Changing variable in the first integral in $(4.47)$ and using $f(t /(1-\eta)) \leqslant f(t)$, we see that it is finite by Lemma 4.6.

Now we deal with the second integral in (4.47). By (4.44), for all $y>0$,

$$
\bar{\Pi}(\varepsilon)(1-\rho(\varepsilon, y))=\bar{\Pi}(\varepsilon)\left(1-\exp \left(-\frac{A_{+}(y)}{64 y \bar{\Pi}(\varepsilon)}\right)\right) \geqslant \frac{A_{+}(y)}{64 y} \exp \left(-\frac{A_{+}(y)}{64 y \bar{\Pi}(\varepsilon)}\right) .
$$

Copyright $@$ ( ) by SIAM. Unauthorized reproduction of this article is prohibited. 
Recall that $\bar{\Pi}(\varepsilon)>1 / x_{0}$. The function $A_{+}(y) / y$ is positive, continuous, and strictly decreasing on $\left(0, x_{0}\right)$, taking value $\infty$ as $y \downarrow 0$ (since $\bar{\Pi}^{+}(0+)=\infty$; see the lines following (4.33)) and value 0 as $y \rightarrow \infty$. So we can define a unique $y(\varepsilon)$ such that

$$
\frac{A_{+}(y(\varepsilon))}{y(\varepsilon)}=\bar{\Pi}(\varepsilon)
$$

Then $y(\varepsilon) \in(0, \infty)$ and $y(\varepsilon) \downarrow 0$ as $\varepsilon \downarrow 0$. Assume $\varepsilon$ is also small enough for $y(\varepsilon) \leqslant x_{0}$. We have to consider two subcases.

Case (a)(i). Suppose $y(\varepsilon) \leqslant \varepsilon$. Observe that $y>y(\varepsilon)$ implies $A_{+}(y) / y \leqslant$ $A_{+}(y(\varepsilon)) / y(\varepsilon)=\bar{\Pi}(\varepsilon)$, and hence by $(4.48)$

$$
\bar{\Pi}(\varepsilon)(1-\rho(\varepsilon, y)) \geqslant \frac{e^{-1 / 64} A_{+}(y)}{64 y} \geqslant \frac{A_{+}(y)}{65 y} .
$$

Choose $y_{0}>0$ such that $y / A_{+}(y) \leqslant x_{0}$ for $0<y \leqslant y_{0}$. Then the second integral in (4.47) does not exceed

$$
\begin{aligned}
& \int_{y(\varepsilon)<y \leqslant 1} \int_{0}^{1} f(t) e^{-t \bar{\Pi}(\varepsilon)(1-\rho(\varepsilon, y))} \mathrm{d} t \Pi^{(-)}(\mathrm{d} y) \\
& \leqslant \int_{y(\varepsilon)<y \leqslant y_{0}} \int_{0}^{1} f(t) e^{-t A_{+}(y) / 65 y} \mathrm{~d} t \Pi^{(-)}(\mathrm{d} y)+\int_{y_{0}<y \leqslant 1} \int_{0}^{1} f(t) \mathrm{d} t \Pi^{(-)}(\mathrm{d} y) \\
& \leqslant \int_{y(\varepsilon)<y \leqslant y_{0}}\left(\frac{y}{A_{+}(y)}\right) \int_{0}^{A_{+}(y) / y} f\left(\frac{t y}{A_{+}(y)}\right) e^{-t / 65} \mathrm{~d} t \Pi^{(-)}(\mathrm{d} y)+c_{++} \bar{\Pi}\left(y_{0}\right)
\end{aligned}
$$

(using (4.23)). Write the inner integral in (4.51) as

$$
\left[\int_{0}^{1}+\int_{1}^{A_{+}(y) / y}\right] f\left(\frac{t y}{A_{+}(y)}\right) e^{-t / 65} \mathrm{~d} t .
$$

In the first of these integrals, $0<t \leqslant 1$ and $y / A_{+}(y) \leqslant x_{0}<1$, so $f\left(t y / A_{+}(y)\right) \leqslant$ $c_{+} t^{-\alpha} f\left(y / A_{+}(y)\right)$ by $(2.7)$. In the second integral, $t \geqslant 1$, so $f\left(t y / A_{+}(y)\right) \leqslant f\left(y / A_{+}(y)\right)$ since $f$ is nonincreasing. So the right-hand side of (4.51) is bounded above by $c_{++} \bar{\Pi}\left(y_{0}\right)$ plus

$$
\int_{y(\varepsilon)<y \leqslant 1}\left(\frac{y}{A_{+}(y)}\right) f\left(\frac{y}{A_{+}(y)}\right)\left[\int_{0}^{\infty}\left(c_{+} t^{-\alpha}+1\right) e^{-t / 65} \mathrm{~d} t\right] \Pi^{(-)}(\mathrm{d} y),
$$

and the last expression is finite as $\varepsilon \downarrow 0$ by assumption (2.11), which implies (4.28).

Case (a)(ii). Suppose $y(\varepsilon)>\varepsilon$. Assume at first that $f(0+)<\infty$. Then the second integral in (4.47) does not exceed $f(0+)$ times

$$
\begin{aligned}
\int_{\varepsilon<y \leqslant 1} & \int_{0}^{x_{0}} e^{-t \bar{\Pi}(\varepsilon)(1-\rho(\varepsilon, y))} \mathrm{d} t \Pi^{(-)}(\mathrm{d} y) \\
& \leqslant\left[\int_{\varepsilon<y \leqslant y(\varepsilon)}+\int_{y(\varepsilon)<y \leqslant 1}\right] \frac{\Pi^{(-)}(\mathrm{d} y)}{\bar{\Pi}(\varepsilon)(1-\rho(\varepsilon, y))} .
\end{aligned}
$$

When $0<y \leqslant y(\varepsilon), A_{+}(y) / y \geqslant A_{+}(y(\varepsilon)) / y(\varepsilon)=\bar{\Pi}(\varepsilon)$, so by (4.44),

$$
1-\rho(\varepsilon, y) \geqslant 1-e^{-1 / 64} \text {. }
$$

Copyright (c) by SIAM. Unauthorized reproduction of this article is prohibited. 
When $y>y(\varepsilon),(4.50)$ applies. Thus the right-hand side of (4.53) does not exceed

$$
\frac{1}{\bar{\Pi}(\varepsilon)\left(1-e^{-1 / 64}\right)} \int_{\varepsilon<y \leqslant 1} \Pi^{(-)}(\mathrm{d} y)+65 \int_{0}^{1}\left(\frac{y}{A_{+}(y)}\right) \Pi^{(-)}(\mathrm{d} y) .
$$

The first term on the right-hand side is smaller than $\left(1-e^{-1 / 64}\right)^{-1}$, and the second is finite by (4.28) with $f \equiv 1$.

Alternatively, $f(0+)=\infty$, in which case $f$ satisfies (2.8) with $\beta>0$. Recall $\bar{\Pi}(\varepsilon)>1$. In this case use Chebyshev's rather than Bernstein's inequality in (4.43) to obtain

$$
\begin{aligned}
\mathbf{P}\left(S_{n}^{y}(\varepsilon)-\mathbf{E} S_{n}^{y}(\varepsilon) \leqslant-u\right) & \leqslant \frac{n U(y)}{u^{2} \bar{\Pi}(\varepsilon)} \leqslant \frac{3 n y A(y)}{u^{2} \bar{\Pi}(\varepsilon)} \quad(\text { by }(4.5)) \\
& =\frac{12 \bar{\Pi}(\varepsilon) y}{n A(y)} \leqslant\left(\frac{24 \bar{\Pi}(\varepsilon)}{n+1}\right)\left(\frac{y}{A(y)}\right),
\end{aligned}
$$

correct for $n=0,1, \ldots$ For $0<y \leqslant y_{0}$ we can replace $A(y)$ by $A_{+}(y)$ in this if we replace 24 by 48 . When $0<y \leqslant y(\varepsilon)$ we have $A_{+}(y) / y \geqslant A_{+}(y(\varepsilon)) / y(\varepsilon)=\bar{\Pi}(\varepsilon)$.

Now go back to (4.39) and split the integral over $\left(\varepsilon, b_{n+1}(\varepsilon)\right]$ into integrals over $(\varepsilon, y(\varepsilon)]$ and $\left(y(\varepsilon), b_{n+1}(\varepsilon)\right.$ ]. (If $y(\varepsilon)>b_{n+1}(\varepsilon)$, simply omit the second integral.) Use the bound in (4.54) in the first of these and the same calculation as in (4.45) for the second to write, as an upper bound for (4.39),

$$
\begin{aligned}
& t e^{-t \bar{\Pi}(\varepsilon)} \sum_{n \geqslant 0} \frac{(t \bar{\Pi}(\varepsilon))^{n}}{n !} \int_{\varepsilon<y \leqslant y(\varepsilon)}\left(\frac{48 \bar{\Pi}(\varepsilon)}{n+1}\right)\left(\frac{y}{A_{+}(y)}\right) \Pi^{(-)}(\mathrm{d} y) \\
& \quad+t \int_{y(\varepsilon)<y \leqslant 1} e^{-t \bar{\Pi}(\varepsilon)(1-\rho(\varepsilon, y))} \Pi^{(-)}(\mathrm{d} y) .
\end{aligned}
$$

As required in (4.46), when multiplied by $t^{-1} f(t)$ and integrated over $0<t \leqslant x_{0}$, the second of these integrals is bounded by the left-hand side of (4.51), and hence finite as $\varepsilon \downarrow 0$ just as in (4.52). The first term in (4.55) is

$$
\begin{array}{r}
48 e^{-t \bar{\Pi}(\varepsilon)} \sum_{n \geqslant 0} \frac{(t \bar{\Pi}(\varepsilon))^{n+1}}{(n+1) !} \int_{\varepsilon<y \leqslant y(\varepsilon)}\left(\frac{y}{A_{+}(y)}\right) \Pi^{(-)}(\mathrm{d} y) \\
=48\left(1-e^{-t \bar{\Pi}(\varepsilon)}\right) \int_{\varepsilon<y \leqslant y(\varepsilon)}\left(\frac{y}{A_{+}(y)}\right) \Pi^{(-)}(\mathrm{d} y) .
\end{array}
$$

Use $y / A_{+}(y) \leqslant 1 / \bar{\Pi}(\varepsilon)$, so $f\left(y / A_{+}(y)\right) \geqslant f(1 / \bar{\Pi}(\varepsilon))$, to bound the last integral by

$$
\frac{48}{f(1 / \bar{\Pi}(\varepsilon))}\left(1-e^{-t \bar{\Pi}(\varepsilon)}\right) \int_{0<y \leqslant 1}\left(\frac{y}{A_{+}(y)}\right) f\left(\frac{y}{A_{+}(y)}\right) \Pi^{(-)}(\mathrm{d} y) .
$$

We have to multiply this by $t^{-1} f(t)$ and integrate over $0<t \leqslant x_{0}$. So we need to bound

$$
\begin{aligned}
& \frac{1}{f(1 / \bar{\Pi}(\varepsilon))} \int_{0}^{x_{0}} t^{-1} f(t)\left(1-e^{-t \bar{\Pi}(\varepsilon)}\right) \mathrm{d} t \\
& \quad=\int_{0}^{x_{0} \bar{\Pi}(\varepsilon)} t^{-1} \frac{f(t / \bar{\Pi}(\varepsilon))}{f(1 / \bar{\Pi}(\varepsilon))}\left(1-e^{-t}\right) \mathrm{d} t .
\end{aligned}
$$

Copyright (c) by SIAM. Unauthorized reproduction of this article is prohibited. 
Now (2.8) (with $\beta>0$, since $f(0+)=\infty$ ) implies

$$
\frac{f(\mu y)}{f(y)} \leqslant \frac{\mu^{-\beta}}{c_{-}}
$$

for all $\mu \geqslant 1,0<y \leqslant x_{0} / \mu$. So $1 \leqslant t \leqslant x_{0} \bar{\Pi}(\varepsilon)$ implies $1 / \bar{\Pi}(\varepsilon) \leqslant x_{0} / t$ and

$$
\frac{f(t / \bar{\Pi}(\varepsilon))}{f(1 / \bar{\Pi}(\varepsilon))} \leqslant \frac{t^{-\beta}}{c_{-}} .
$$

Using this, together with (2.7), gives an upper bound for the right-hand side of (4.57) as

$$
\left[c_{+} \int_{0}^{1} t^{-1-\alpha}+\int_{1}^{\infty} t^{-1-\beta} / c_{-}\right]\left(1-e^{-t}\right) \mathrm{d} t
$$

and since $\alpha<1$, this is finite, so again we have a finite upper bound. We have proved that the integral in (2.10) is finite when (2.11) holds, in the case $\mathrm{d}_{X}=0$.

Case (b). In the alternative case, $\mathrm{d}_{X}>0$, we have $\lim _{y \downarrow 0} A(y)=\mathrm{d}_{X}$. To check for convergence, the integral in (2.11) can be replaced by the same integral with $A(y)$ simply omitted (replaced by 1 ). This follows from the monotonicity of $f$ and (2.7) and (2.8). Modify the proof of Case (a) by setting $y(\varepsilon)=1 / \bar{\Pi}(\varepsilon)$ and replacing $\rho(\varepsilon, y)$ in (4.44) by

$$
\widetilde{\rho}(\varepsilon, y)=\exp \left(-\frac{1}{64 y \bar{\Pi}(\varepsilon)}\right) .
$$

The argument in (4.45)-(4.49) remains valid with $\rho(\varepsilon, y)$ and $A_{+}(y)$ replaced by $\widetilde{\rho}(\varepsilon, y)$ and 1 , so the proof can be carried through as before.

Case (b)(i). We suppose that $y(\varepsilon)=1 / \bar{\Pi}(\varepsilon) \leqslant \varepsilon$. For $y>y(\varepsilon)$, so $1 / y \leqslant \bar{\Pi}(\varepsilon)$, replace (4.50) by

$$
\bar{\Pi}(\varepsilon)(1-\widetilde{\rho}(\varepsilon, y)) \geqslant \frac{e^{-1 / 64}}{64 y} \geqslant \frac{1}{65 y} .
$$

Then the bounds in (4.51) and (4.52) remain valid if $y(\varepsilon)$ and $A_{+}(y)$ are replaced by $1 / \bar{\Pi}(\varepsilon)$ and 1. The modified bound (4.52) is finite under (2.11) with $A(y)$ replaced by 1 .

Case (b)(ii). Suppose $1>1 / \bar{\Pi}(\varepsilon)>\varepsilon$. Relations (4.5) remains valid when $\lim _{y \downarrow 0} A(y)=\mathrm{d}_{X}$ since

$$
U(x)=2 \int_{0}^{x} y \bar{\Pi}(y) \mathrm{d} y \leqslant 2 x \int_{0}^{x} \bar{\Pi}(y) \mathrm{d} y=o(x) \quad \text { as } x \downarrow 0
$$

(since $X \in b v$ ). So (4.43) remains valid for small $y$ if $A(y)$ is replaced by 1 . Then we get (4.56) with $A_{+}(y)$ replaced by 1 . Again this gives a finite quantity. Lemma 4.8 is proved.

Lemma 4.9. Suppose (2.11) holds. Then

$$
\sum_{n \geqslant 0} \mathbf{P}\left(X_{2^{-n}} \leqslant 0\right)<\infty .
$$

Copyright (c) by SIAM. Unauthorized reproduction of this article is prohibited. 
Proof of Lemma 4.9. Assume (2.11) holds, so by Lemma 4.7, $X \in b v$. Set $f \equiv 1$. obtain

Case (a). We have $\mathrm{d}_{X}=0$. Use the estimates in (4.36), (4.37), and (4.45) to

$$
\begin{aligned}
\mathbf{P}\left(X_{2^{-n}} \leqslant 0\right) \leqslant 2^{-n+1} \bar{\Pi}^{-}\left(b\left((1-\eta) 2^{-n}\right)\right) & \\
& +2^{-n} \limsup _{\varepsilon \downarrow 0} \int_{\varepsilon<y \leqslant 1} e^{-2^{-n} \bar{\Pi}(\varepsilon)(1-\rho(\varepsilon, y))} \Pi^{(-)}(\mathrm{d} y) .
\end{aligned}
$$

Summing over $n$ we get for the first term on the right-hand side of (4.59)

$$
\begin{aligned}
& \sum_{n \geqslant 1} 2^{-n-1} \bar{\Pi}^{-}\left(b\left((1-\eta) 2^{-n}\right)\right) \\
& \quad=\sum_{n \geqslant 1} 2^{-n-1} \sum_{j=1}^{n} \int_{b\left((1-\eta) 2^{-j}\right)<x \leqslant b\left((1-\eta) 2^{-j+1}\right)} \Pi^{(-)}(\mathrm{d} x)+C \\
& \quad \leqslant 2 \sum_{j \geqslant 1} 2^{-j} \int_{b\left((1-\eta) 2^{-j}\right)<x \leqslant b\left((1-\eta) 2^{-j+1}\right)} \Pi^{(-)}(\mathrm{d} x)+C \\
& \quad \leqslant 2 \int_{(0, b(1-\eta)]} b^{\leftarrow}(x /(1-\eta)) \Pi^{(-)}(\mathrm{d} x)+C .
\end{aligned}
$$

(Here $C:=\bar{\Pi}^{-}(b(1-\eta))$ and we keep $\eta \in(0,1 / 2)$.) When (2.11) holds, this is finite as shown in Lemma 4.6 (with $f \equiv 1$ ). To deal with the second term on the right-hand side of (4.59), use the inequality

$$
\sum_{n \geqslant 0} 2^{-n} e^{-2^{-n} a} \leqslant \frac{2}{a}
$$

valid for $a>0$, to write

$$
\sum_{n \geqslant 0} 2^{-n} \int_{\varepsilon<y \leqslant 1} e^{-2^{-n} \bar{\Pi}(\varepsilon)(1-\rho(\varepsilon, y))} \Pi^{(-)}(\mathrm{d} y) \leqslant 2 \int_{\varepsilon<y \leqslant 1} \frac{\Pi^{(-)}(\mathrm{d} y)}{\bar{\Pi}(\varepsilon)(1-\rho(\varepsilon, y))} .
$$

Define $y(\varepsilon)$ to satisfy (4.49), and suppose $y(\varepsilon) \leqslant \varepsilon$. When $y>y(\varepsilon),(4.50)$ holds and

$$
\int_{\varepsilon<y \leqslant 1} \frac{\Pi^{(-)}(\mathrm{d} y)}{\bar{\Pi}(\varepsilon)(1-\rho(\varepsilon, y))} \leqslant \int_{y(\varepsilon)<y \leqslant 1} \leqslant 65 \int_{0}^{1}\left(\frac{y}{A_{+}(y)}\right) \Pi^{(-)}(\mathrm{d} y)
$$

is finite by (4.28). Alternatively, when $y(\varepsilon)>\varepsilon$, the right-hand side of (4.60) is finite as in (4.53) and the following.

Case (b). The case when $d_{X}>0$ is handled similarly as in Lemma 4.8. Thus we get (4.58). Lemma 4.9 is proved.

Lemma 4.10. Suppose (2.11) holds. Then $\mathrm{d}_{X}>0$ or $\mathrm{d}_{X}=0$ and

$$
\lim _{t \downarrow 0} \frac{\sum_{0<s \leqslant t} \Delta X_{s}^{-}}{\sum_{0<s \leqslant t} \Delta X_{s}^{+}}=0 \quad \text { a.s. }
$$

Proof of Lemma 4.10. Assume (2.11) holds. Then $X \in b v$ and $\mathrm{d}_{X} \geqslant 0$ by Lemma 4.7, and $\sum \mathbf{P}\left(X_{2^{-n}} \leqslant 0\right)<\infty$ by Lemma 4.9. From the Borel-Cantelli lemma we deduce $\mathbf{P}\left(X_{2^{-n}} \leqslant 0\right.$ i.o. $)=0$. If $\mathrm{d}_{X}=0$, then (4.34) shows that 
$\sum_{0<s \leqslant 2^{-n}} \Delta X_{s}^{-} \leqslant \sum_{0<s \leqslant 2^{-n}} \Delta X_{s}^{+}$all but finitely often, a.s., as $n \rightarrow \infty$. Given $t \in(0,1)$ let $n=n(t)=\left\lfloor-\log _{2} t\right\rfloor$. Then

$$
\begin{aligned}
\frac{\sum_{0<s \leqslant t} \Delta X_{s}^{-}}{\sum_{0<s \leqslant t} \Delta X_{s}^{+}} & \leqslant \frac{\sum_{0<s \leqslant 2^{-n}} \Delta X_{s}^{-}}{\sum_{0<s \leqslant 2^{-n-1}} \Delta X_{s}^{+}} \\
& =\frac{\sum_{0<s \leqslant 2^{-n-1}} \Delta X_{s}^{-}}{\sum_{0<s \leqslant 2^{-n-1}} \Delta X_{s}^{+}}+\frac{\sum_{2^{-n-1}<s \leqslant 2^{-n}} \Delta X_{s}^{-}}{\sum_{0<s \leqslant 2^{-n-1}} \Delta X_{s}^{+}} .
\end{aligned}
$$

The first term on the right-hand side has limsup $\leqslant 1$ a.s. By independence of $\left(\Delta X_{t}^{+}\right)_{t \geqslant 0}$ and $\left(\Delta X_{t}^{-}\right)_{t \geqslant 0}$, and the stationarity of $\left(\Delta X_{t}^{-}\right)_{t \geqslant 0}$,

$$
\sum_{n \geqslant 0} \mathbf{P}\left(\sum_{2^{-n-1}<s \leqslant 2^{-n}} \Delta X_{s}^{-} \geqslant \sum_{0<s \leqslant 2^{-n-1}} \Delta X_{s}^{+}\right)=\sum_{n \geqslant 0} \mathbf{P}\left(X_{2^{-n-1}} \leqslant 0\right) .
$$

The right-hand side here is finite, so the second term also has limsup $\leqslant 1$ a.s., and we get

$$
\limsup _{t \downarrow 0} \frac{\sum_{0<s \leqslant t} \Delta X_{s}^{-}}{\sum_{0<s \leqslant t} \Delta X_{s}^{+}} \leqslant 2 \quad \text { a.s. }
$$

By considering the Lévy process $X_{t}^{a}:=a \sum_{0<s \leqslant t} \Delta X_{s}^{+}-\sum_{0<s \leqslant t} \Delta X_{s}^{-}$, with $0<$ $a<1$, in place of $X$, it is not hard to see that (4.62) can be improved to (4.61). Lemma 4.10 is proved.

Lemma 4.11. Suppose $X \in$ bv with drift $\mathrm{d}_{X}=0$ and $\bar{\Pi}^{+}(0+)=\infty$. Then (4.61) implies (4.28).

Proof of Lemma 4.11. Assume $X \in b v, d_{X}=0$, and (4.61) holds. Take $a>1$ and write

$$
X_{t}^{a}:=a \sum_{0<s \leqslant t} \Delta X_{s}^{+}-\sum_{0<s \leqslant t} \Delta X_{s}^{-}=: X_{t}^{a,+}-X_{t}^{a,-} .
$$

$X_{t}^{a}$ is bv with drift 0 and jumps $\Delta X_{t}^{a,+}=a \Delta X_{s}^{+}$and $\Delta X_{t}^{a,-}=\Delta X_{s}^{-}$, and by (4.61),

$$
\mathbf{P}\left(X_{t}^{a} \leqslant 0 \text { i.o. as } t \downarrow 0\right)=\mathbf{P}\left(\frac{\sum_{0<s \leqslant t} \Delta X_{s}^{-}}{\sum_{0<s \leqslant t} \Delta X_{s}^{+}} \geqslant a \text { i.o. as } t \downarrow 0\right)=0 .
$$

(Since $\bar{\Pi}^{+}(0+)=\infty$, the denominator here is positive a.s.) Suppose (4.28) fails to hold. Then since $f(0+)>0$, it fails to hold with $f \equiv 1$, i.e., (4.31) fails to hold. Then for a contradiction we want to show $\mathbf{P}\left(X_{t}^{a} \leqslant 0\right.$ i.o. as $\left.t \downarrow 0\right)>0$. Given $\eta \in(0,1)$, choose $\delta>0$ so that $21 a \delta<\eta(1-2 \delta a)^{2}$. Define the function

$$
c(t):=c_{\delta}(t)=\inf \left\{0<x \leqslant x_{0}: \frac{A_{+}(x)}{x} \leqslant \frac{\delta}{t}\right\}, \quad t>0,
$$

where $A_{+}(x)>0$ for $0<x \leqslant x_{0}$ since $\bar{\Pi}^{+}(0+)=\infty$. Then $c(t)$ is continuous and strictly increasing and satisfies

$$
\frac{A_{+}(c(t))}{c(t)}=\frac{\delta}{t}, \quad t>0 .
$$

Copyright $@$ ( ) by SIAM. Unauthorized reproduction of this article is prohibited. 
Assume, for some $t_{n} \downarrow 0$, we have

$$
\limsup _{n \rightarrow \infty} \mathbf{P}\left(X_{t_{n}}^{a,+}>\frac{c\left(t_{n}\right)}{2}\right) \leqslant \eta
$$

and

$$
\mathbf{P}\left(X_{t_{n}}^{a,-}>c\left(t_{n}\right) \text { i.o. as } n \rightarrow \infty\right)=1 .
$$

Then by the Feller-Chung inequality (see [8, p. 70])

$$
\begin{aligned}
\mathbf{P}\left(X_{t}^{a}\right. & \leqslant 0 \text { i.o. as } t \downarrow 0) \geqslant \mathbf{P}\left(X_{t_{n}}^{a,-} \geqslant X_{t_{n}}^{a,+} \text { i.o. as } n \rightarrow \infty\right) \\
& \geqslant \lim _{m \rightarrow \infty} \mathbf{P}\left(X_{t_{n}}^{a,-}>c\left(t_{n}\right), X_{t_{n}}^{a,+} \leqslant \frac{c\left(t_{n}\right)}{2} \text { for some } n>m\right) \\
& \geqslant(1-\eta) \mathbf{P}\left(X_{t_{n}}^{a,-}>c\left(t_{n}\right) \text { i.o. as } n \rightarrow \infty\right) \geqslant 1-\eta .
\end{aligned}
$$

Hence it is enough to show (4.65) and (4.66) with $t_{n}=2^{-n}$.

To prove (4.65), observe that, since $a>1$,

$$
\begin{aligned}
& \mathbf{E}\left(\sum_{0<s \leqslant t}\left(\Delta X_{s}^{a,+} \wedge c(t)\right)\right)=\mathbf{E}\left(\sum_{0<s \leqslant t}\left(a \Delta X_{s}^{+} \wedge c(t)\right)\right) \\
& =t A_{+}^{a}(c(t))=a t A_{+}\left(\frac{c(t)}{a}\right) \leqslant a t A_{+}(c(t))=\delta a c(t) .
\end{aligned}
$$

Consequently, we have

$$
\begin{aligned}
& \mathbf{P}\left(X_{t}^{a,+}>\frac{c(t)}{2}\right) \leqslant \mathbf{P}\left(a \Delta X_{s}^{+}>c(t) \text { for some } s \leqslant t\right) \\
& \quad+\mathbf{P}\left(\sum_{0<s \leqslant t}\left(\Delta X_{s}^{a,+} \wedge c(t)\right)-\mathbf{E} \sum_{0<s \leqslant t}\left(\Delta X_{s}^{a,+} \wedge c(t)\right)>\frac{c(t)}{2}-\delta a c(t)\right) \\
& \leqslant t \bar{\Pi}^{+}\left(\frac{c(t)}{a}\right)+\frac{4 \operatorname{Var}\left(\sum_{0<s \leqslant t}\left(\Delta X_{s}^{a,+} \wedge c(t)\right)\right)}{c^{2}(t)(1-2 \delta a)^{2}}
\end{aligned}
$$

in which

$$
\operatorname{Var}\left(\sum_{0<s \leqslant t}\left(\Delta X_{s}^{a,+} \wedge c(t)\right)\right) \leqslant t a^{2} \int_{0}^{c(t) / a} x \bar{\Pi}^{+}(x) \mathrm{d} x \leqslant t a^{2} U\left(\frac{c(t)}{a}\right) .
$$

So, when $0<t<a / x_{0}$, the right-hand side of (4.68) is not greater than

$$
t \bar{\Pi}^{+}\left(\frac{c(t)}{a}\right)+\left(\frac{4}{(1-2 \delta a)^{2}}\right) \frac{t a^{2} U(c(t) / a)}{c^{2}(t)} \leqslant\left(\frac{7}{(1-2 \delta a)^{2}}\right) \frac{t a^{2} U(c(t))}{c^{2}(t)}
$$

since $x^{2} \bar{\Pi}(x) \leqslant U(x)$ and $a>1$. Now (4.61) implies (4.2), so we have (4.5). Thus the last expression is not greater than

$$
\left(\frac{7}{(1-2 \delta a)^{2}}\right) \frac{3 t a A(c(t))}{c(t)} \leqslant\left(\frac{7}{(1-2 \delta a)^{2}}\right) 3 a \delta<\eta,
$$

for small $t$, by the choice of $\delta$. This proves (4.65), with $t_{n}$ replaced by $t$.

Copyright $($ C by SIAM. Unauthorized reproduction of this article is prohibited. 
To prove (4.66), start by noticing that

$$
X_{2^{-n}}^{a,-}=\sum_{0<t \leqslant 2^{-n}} \Delta X_{t}^{-} \geqslant \sup _{2^{-n-1}<t \leqslant 2^{-n}} \Delta X_{t}^{-} .
$$

Since the events involved are independent,

$$
\begin{array}{r}
\mathbf{P}\left(\sup _{2^{-n-1}<t \leqslant 2^{-n}} \Delta X_{t}^{-}>c\left(2^{-n}\right) \text { i.o. }\right)=1 \quad \text { if and only if } \\
\sum_{n \geqslant 0} \mathbf{P}\left(\sup _{2^{-n-1}<t \leqslant 2^{-n}} \Delta X_{t}^{-}>c\left(2^{-n}\right)\right)=\infty .
\end{array}
$$

Relation (4.61) implies (4.2), so by (4.3), $x \bar{\Pi}^{-}(x)=o(A(x))=o\left(A_{+}(x)\right)$ as $x \downarrow 0$. Hence

$$
t \bar{\Pi}^{-}(c(t))=o\left(\frac{c(t) \bar{\Pi}^{-}(c(t))}{A_{+}(c(t))}\right) \rightarrow 0 .
$$

The sum on the right-hand side of (4.69) is

$$
\sum_{n \geqslant 0}\left(1-e^{-2^{-n-1} \bar{\Pi}^{-}\left(c\left(2^{-n}\right)\right)}\right) \geqslant \sum_{n \geqslant 0} e^{-(1+o(1))} 2^{-n-1} \bar{\Pi}^{-}\left(c\left(2^{-n}\right)\right) .
$$

The function $c(x)$ has a unique inverse function $\delta x / A_{+}(x)$ (see (4.64)), so

$$
\sum_{n \geqslant 1} 2^{-n} \bar{\Pi}^{-}\left(c\left(2^{-n}\right)\right) \geqslant \sum_{n \geqslant 1} \int_{2^{-n}}^{2^{-n+1}} \bar{\Pi}^{-}(c(x)) \mathrm{d} x=\delta \int_{0}^{c(1)} \bar{\Pi}^{-}(x) \mathrm{d}\left(\frac{x}{A_{+}(x)}\right) .
$$

The right-hand side is the integral in (1.2) and is infinite when (4.31) fails to hold, as can be seen by integrating by parts. Thus $\mathbf{P}\left(X_{2^{-n}}^{a,-}>c\left(2^{-n}\right)\right.$ i.o. as $\left.n \rightarrow \infty\right)=1$, and we have (4.66) with $t_{n}=2^{-n}$. Relation (4.67) gives the contradiction required to prove the lemma. Lemma 4.11 is proved.

\section{Proof of Theorem 2.1.}

Lemma 5.1. Suppose (2.7) and (2.8) hold. Then (2.10) is equivalent to (2.11).

Proof of Lemma 5.1. Assume (2.7), (2.8), and (2.10) hold. Since $f(0+)>0,(2.10)$ implies the convergence of the integral in (1.1), so by Lemma 4.1, we have $\sigma^{2}=0$ and (4.2). Thus (4.5)-(4.7) hold, and we can define $b(\cdot) \in\left(0, x_{0}\right]$ by (4.8) with $0<\delta<1 / 12$ and $x_{0}$ as in (4.5). Then by Lemma 4.5, $16 \mathbf{P}\left(X_{t} \leqslant 0\right) \geqslant t \bar{\Pi}^{-}(b(t+))$, so $\int_{0}^{1} f(t) \bar{\Pi}^{-}(b(t+)) \mathrm{d} t$ is finite by (2.10). This implies convergence of the integral in (2.11) by Lemma 4.6. In the other direction, assume (2.7), (2.8), and (2.11). Then (2.10) holds by Lemma 4.7. Lemma 5.1 is proved.

To complete Theorem 2.1 we add in the finiteness of the moment condition for $T_{0}^{-}$. As a result of the definition $T_{0}^{-}:=\inf \left\{t>0: X_{t}<0\right\}$, we have

$$
\mathbf{P}\left(T_{0}^{-}<t\right) \leqslant \mathbf{P}\left(\underline{X}_{t}<0\right) \leqslant \mathbf{P}\left(T_{0}^{-} \leqslant t\right)
$$

where $\underline{X}_{t}:=\inf _{0 \leqslant s \leqslant t} X_{s}, t \geqslant 0$. The following lemma, a consequence of the WienerHopf factorization, connects $\mathbf{P}\left(X_{t}<0\right)$ and $\mathbf{P}\left(\underline{X}_{t}<0\right)$.

Copyright (c) by SIAM. Unauthorized reproduction of this article is prohibited. 
Lemma 5.2. For any $0<p<\infty$,

$$
1-p \int_{0}^{\infty} e^{-p t} \mathbf{P}\left(\underline{X}_{t}<0\right) \mathrm{d} t=\exp \left\{-\int_{0}^{\infty} e^{-p t} \mathbf{P}\left(X_{t}<0\right) \frac{\mathrm{d} t}{t}\right\}
$$

and

$$
p \int_{0}^{\infty} e^{-p t} \mathbf{P}\left(\underline{X}_{t}<0\right) \mathrm{d} t \leqslant \int_{0}^{\infty} e^{-p t} \mathbf{P}\left(X_{t}<0\right) \frac{\mathrm{d} t}{t} .
$$

Proof of Lemma 5.2. Equation (5.2) follows from [19, eq. (47.10)] applied to $\left(-X_{t}\right)_{t \geqslant 0}$ on noting that $\mathbf{P}\left(\underline{X}_{t} \leqslant 0\right)=1$. Take the logarithm of both sides of (5.2) and use the inequality $z<-\ln (1-z), 0<z<1$, to get (5.3). Lemma 5.1 is proved.

Lemma 5.3. Assume (2.6)-(2.9) and $\int_{1}^{\infty} t^{-1} f(t) \mathrm{d} t<\infty$. Then (2.10) is equivalent to (2.12).

Proof of Lemma 5.3. Assume (2.6)-(2.9) and $\int_{1}^{\infty} t^{-1} f(t) \mathrm{d} t<\infty$. When $f(0+)<$ $\infty$ we deduce $T_{0}^{-}>0$ a.s. by (1.1). When $f(0+)=\infty$ we have by (2.9)

$$
\begin{aligned}
\infty & >\int_{0}^{\infty} t^{-1} f(t) \mathbf{P}\left(X_{t}<0\right) \mathrm{d} t=\int_{0}^{\infty} t^{-1} \int_{0}^{\infty} e^{-p t} \mu(\mathrm{d} p) \mathbf{P}\left(X_{t}<0\right) \mathrm{d} t \\
& \geqslant \int_{0}^{\infty} p \int_{0}^{\infty} e^{-p t} \mathbf{P}\left(\underline{X}_{t}<0\right) \mathrm{d} t \mu(\mathrm{d} p) \quad(\text { by }(5.3)) \\
& \geqslant \int_{0}^{\infty} p \int_{0}^{\infty} e^{-p t} \mu(\mathrm{d} p) \mathbf{P}\left(T_{0}^{-}<t\right) \mathrm{d} t \quad(\text { by }(5.1)) \\
& =\int_{0}^{\infty} \int_{0}^{\infty} e^{-p t} \mu(\mathrm{d} p) \mathbf{P}\left(T_{0}^{-} \in \mathrm{d} t\right)=\mathbf{E} f\left(T_{0}^{-}\right),
\end{aligned}
$$

where for the second equality we integrated by parts and used (2.9) again. So we have proved that (2.10) implies (2.12).

In the other direction, assume $\mathbf{E} f\left(T_{0}^{-}\right)<\infty$, so $T_{0}^{-}>0$ a.s. By (2.9) and (5.2),

$$
\begin{aligned}
\int_{0}^{\infty} t^{-1} f(t) \mathbf{P}\left(X_{t}<0\right) \mathrm{d} t & =\int_{0}^{\infty} t^{-1} \int_{0}^{\infty} e^{-p t} \mu(\mathrm{d} p) \mathbf{P}\left(X_{t}<0\right) \mathrm{d} t \\
& =-\int_{0}^{\infty} \ln \left(1-p \int_{0}^{\infty} e^{-p t} \mathbf{P}\left(\underline{X}_{t}<0\right) \mathrm{d} t\right) \mu(\mathrm{d} p) \\
& \leqslant-\int_{0}^{\infty} \ln \left(1-\mathbf{E}\left(e^{-p T_{0}^{-}}\right)\right) \mu(\mathrm{d} p) \\
& =\int_{p=0}^{\infty} \int_{u=g(p)}^{1} \frac{\mathrm{d} u}{u} \mu(\mathrm{d} p),
\end{aligned}
$$

where $g(p):=1-\mathbf{E}\left(\exp \left(-p T_{0}^{-}\right)\right), p>0$, and we used $\mathbf{P}\left(\underline{X}_{t}<0\right) \leqslant \mathbf{P}\left(T_{0}^{-} \leqslant t\right)$, implying

$$
\begin{aligned}
p \int_{0}^{\infty} e^{-p t} \mathbf{P}\left(\underline{X}_{t}<0\right) \mathrm{d} t & \leqslant p \int_{0}^{\infty} e^{-p t} \mathbf{P}\left(T_{0}^{-} \leqslant t\right) \mathrm{d} t \\
& =\int_{0}^{\infty} e^{-p t} \mathbf{P}\left(T_{0}^{-} \in \mathrm{d} t\right)=\mathbf{E}\left(e^{-p T_{0}^{-}}\right) .
\end{aligned}
$$

The function $g(\cdot)$ is continuous and strictly increasing with $g(0)=0$ and $g(\infty)=1$. Let $g^{\leftarrow}(\cdot)$ be its inverse function. Change the order of integration, so the right-hand

Copyright (c) by SIAM. Unauthorized reproduction of this article is prohibited. 
side of (5.4) equals

$$
\int_{u=0}^{1} \int_{p=0}^{g^{\leftarrow}(u)} \mu(\mathrm{d} p) \frac{\mathrm{d} u}{u}=\int_{0}^{1} \mu\left(g^{\leftarrow}(u)\right) \frac{\mathrm{d} u}{u}
$$

(recall $\mu(0)=0)$. Also note that

$$
\begin{aligned}
\mathbf{E} f\left(T_{0}^{-}\right) & =\int_{t>0} f(t) \mathbf{P}\left(T_{0}^{-} \in \mathrm{d} t\right)=\int_{y=0}^{\infty} \int_{t>0} e^{-y t} \mathbf{P}\left(T_{0}^{-} \in \mathrm{d} t\right) \mu(\mathrm{d} y) \\
& =\int_{0}^{\infty}(1-g(y)) \mu(\mathrm{d} y)=\int_{0}^{\infty} \mu(y) \mathrm{d} g(y)=\int_{0}^{1} \mu\left(g^{\leftarrow}(z)\right) \mathrm{d} z .
\end{aligned}
$$

So the integral on the right-hand side of (5.6) is finite. We want to deduce from this the finiteness of the integrals in (5.5). Choose $t_{0} \in(0,1)$ and write

$$
\left(\int_{0}^{t_{0}}+\int_{t_{0}}^{1}\right) \mu\left(g^{\leftarrow}(y)\right) \frac{\mathrm{d} u}{u} \leqslant \int_{0}^{t_{0}} \mu\left(g^{\leftarrow}(u)\right) \frac{\mathrm{d} u}{u}+\frac{1}{t_{0}} \int_{0}^{1} \mu\left(g^{\leftarrow}(u)\right) \mathrm{d} u .
$$

The second integral on the right-hand side is finite because of (5.6). It remains to show the same is true of the first one. First, we estimate

$$
\begin{aligned}
\frac{z}{g^{\leftarrow}(z)} & =\frac{1-\mathbf{E}\left(e^{-g^{\leftarrow}(z) T_{0}^{-}}\right)}{g^{\leftarrow}(z)} \\
& =\mathbf{E}\left(\frac{1-e^{-g^{\leftarrow}(z) T_{0}^{-}}}{g^{\leftarrow}(z)}\right) \rightarrow \mathbf{E}\left(T_{0}^{-}\right) \in(0, \infty] \quad \text { as } z \downarrow 0 .
\end{aligned}
$$

Thus $g^{\leftarrow}(z)=O(z)$ as $z \downarrow 0$. Hence, for some $c>0$ and $t_{0}$ small enough,

$$
\int_{0}^{t_{0}} \mu\left(g^{\leftarrow}(u)\right) \frac{\mathrm{d} u}{u} \leqslant \int_{0}^{t_{0}} \mu(c u) \frac{\mathrm{d} u}{u} .
$$

Next, we need an upper bound for $\mu\left(t^{-1}\right)$ of the form

$$
f(t)=\int_{0}^{\infty} e^{-p t} \mu(\mathrm{d} p) \geqslant e^{-1}\left(\mu\left(t^{-1}\right)-\mu(0)\right)=e^{-1} \mu\left(t^{-1}\right), \quad t>0,
$$

showing that

$$
\int_{0}^{t_{0}} \mu(c u) \frac{\mathrm{d} u}{u} \leqslant e \int_{0}^{t_{0}} f\left((c u)^{-1}\right) \frac{\mathrm{d} u}{u}=e \int_{1 / t_{0}}^{\infty} f\left(\frac{u}{c}\right) \frac{\mathrm{d} u}{u} .
$$

By assumption this is finite. Hence the right-hand side of (5.8) is finite, so the same is true for (5.7), (5.5), and (5.4). This proves the lemma and completes the proof of Theorem 2.1. Lemma 5.3 is proved.

Acknowledgments. I am grateful to a referee for suggesting improvements to an earlier draft, and to Yuguang Fan for a careful reading of various versions of the paper.

\section{REFERENCES}

[1] Y. Aїт-Sahalia And J. Jacod, Identifying the successive Blumenthal-Getoor indices of a discretely observed process, Ann. Statist., 40 (2012), pp. 1430-1464.

Copyright (C) by SIAM. Unauthorized reproduction of this article is prohibited. 
[2] J. Bertoin, Lévy Processes, Cambridge Tracts in Math. 121, Cambridge Univ. Press, Cambridge, 1996.

[3] J. Bertoin, Regularity of the half-line for Lévy processes, Bull. Sci. Math., 121 (1997), pp. 345-354.

[4] J. Bertoin And R. A. Doney, Spitzer's condition for random walks and Lévy processes, Ann. Inst. H. Poincaré Probab. Statist., 33 (1997), pp. 167-178.

[5] J. Bertoin, R. A. Doney, And R. A. Maller, Passage of Lévy processes across power law boundaries at small times, Ann. Probab., 36 (2008), pp. 160-197.

[6] N. H. Bingham, C. M. Goldie, and J. L. Teugels, Regular Variation, Encyclopedia Math. Appl. 27, Cambridge Univ. Press, Cambridge, 1987.

[7] R. M. Blumenthal and R. K. Getoor, Sample functions of stochastic processes with stationary independent increments, J. Math. Mech., 10 (1961), pp. 493-516.

[8] Y. S. Chow And H. Teicher, Probability Theory. Independence, Interchangeability, Martingales, 2nd ed., Springer Texts Statist., Springer-Verlag, New York, 1988.

[9] R. A. Doney, Small-time behaviour of Lévy processes, Electron. J. Probab., 9 (2004), pp. 209-229.

[10] R. A. Doney, Fluctuation Theory for Lévy Processes, Lectures from the 35th Summer School on Probability Theory held in Saint-Flour, July 6-23, 2005, edited and with a foreword by Jean Picard, Lecture Notes in Math. 1897, Springer, Berlin, 2007.

[11] P. Erdős, On a theorem of Hsu and Robbins, Ann. Math. Statist., 20 (1949), pp. 286-291.

[12] K. B. Erickson, The strong law of large numbers when the mean is undefined, Trans. Amer. Math. Soc., 185 (1974), pp. 371-381.

[13] W. Feller, An Introduction to Probability Theory and Its Applications, Vol. II, 2nd ed., John Wiley \& Sons, Inc., New York, 1971.

[14] H. Kesten And R. A. Maller, Two renewal theorems for general random walks tending to infinity, Probab. Theory Related Fields, 106 (1996), pp. 1-38.

[15] V. Knopova, R. L. Schilling, And J. Wang, Lower bounds of the Hausdorff dimension for the images of Feller processes, Statist. Probab. Lett., 97 (2015), pp. 222-228. Preprint version available at http://arxiv.org/abs/1406.3849v2 .

[16] A. Kuznetsov, Analytic proof of Pecherskii-Rogozin identity and Wiener-Hopf factorization, Theory Probab. Appl., 55 (2011), pp. 432-443.

[17] B. A. Rogozin, On distributions of functionals related to boundary problems for processes with independent increments, Theory Probab. Appl., 11 (1966), pp. 580-591.

[18] B. A. Rogozin, Local behavior of processes with independent increments, Theory Probab. Appl., 13 (1968), pp. 482-486.

[19] K. Sato, Lévy Processes and Infinitely Divisible Distributions, Cambridge Stud. Adv. Math. 68, Cambridge Univ. Press, Cambridge, 1999.

[20] E. S. Shtatland, On local properties of processes with independent increments, Theory Probab. Appl., 10 (1965), pp. 317-322.

[21] F. Spitzer, A combinatorial lemma and its applications to probability theory, Trans. Amer. Math. Soc., 82 (1956), pp. 323-339.

[22] V. Vigon, Votre Lévy rampe-t-il?, J. London Math. Soc. (2), 65 (2002), pp. 243-256.

Copyright (c) by SIAM. Unauthorized reproduction of this article is prohibited. 